M. Miyake and M. Yoshino

Nagoya Math. J.

Vol. 135 (1994), 165-196

\title{
WIENER-HOPF EQUATION AND FREDHOLM PROPERTY OF THE GOURSAT PROBLEM IN GEVREY SPACE
}

\author{
MASATAKE MIYAKE AND MASAFUMI YOSHINO
}

\section{Dedicated to Professor Yoshio Kato on the occasion of his 60 th birthday}

\section{Introduction}

In the study of ordinary differential equations, Malgrange ([Ma]) and Ramis ([R1], [R2]) established index theorem in (formal) Gevrey spaces, and the notion of irregularity was nicely defined for the study of irregular points. In their studies, a Newton polygon has a great advantage to describe and understand the results in visual form. From this point of view, Miyake ([M2], [M3], [MH]) studied linear partial differential operators on (formal) Gevrey spaces and proved analogous results, and showed the validity of Newton polygon in the study of partial differential equations (see also [Yn]).

The purpose of this paper is to extend results in [M3], where spectral property in Gevrey spaces of a special integro-differential operator was studied, which is induced from the Goursat problem formulated from an interior point of a side of Newton polygon defined by (0.2) below. Precisely, we characterize Fredholm property of the Goursat problem by employing the theory of (finite section) Wiener-Hopf equations, and show that such a property depends deeply on the Hilbert factorizability of Toeplitz symbol associated with the Gevrey index. We note that Fredholm property of the Goursat problem in the category of local holomorphic functions was firstly pointed out by Leray ([L]) by an typical example of operators, and a systematic study of such property is firstly done in this paper.

In order to illustrate our intention, we shall show a typical result which follows from Theorem 1 in Section 1.

Let $P \equiv P\left(t, x ; D_{t}, D_{x}\right)$ be a linear partial differential operator of finite order with holomorphic coefficients in a neighbourhood of the origin of $\mathbf{C}_{t} \times \mathbf{C}_{x}$,

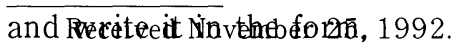




$$
P\left(t, x ; D_{t}, D_{x}\right)=\sum_{\sigma \in \mathbf{N}} \sum_{j, \alpha \in \mathbf{N}}^{\text {finite }} a_{\sigma j \alpha}(\dot{x}) t^{\sigma} D_{t}^{j} D_{x}^{\alpha}
$$

where $\mathbf{N}$ denotes the set of non negative integers. For a triplet $(\sigma, j, \alpha) \in \mathbf{N}^{3}$, we associate a left half line $Q(\sigma, j, \alpha)$ in a $(u, v)$-plane defined by

$$
Q(\sigma, j, \alpha):=\left\{(u, \sigma-j) \in \mathbf{R}^{2} ; u \leq j+\alpha\right\} .
$$

Then a Newton polygon $N(P)$ of the operator $P$ is defined by,

$$
N(P):=\operatorname{ch}\left\{Q(\sigma, j, \alpha) ; a_{\sigma j \alpha}(x) \not \equiv 0\right\},
$$

where $\operatorname{ch}\{\cdot\}$ denotes the convex hull.

For a given $s>0$, we draw a line $L_{s}$ with slope $k:=1 /(s-1) \in \mathbf{R} \cup\{\infty\}$ which contacts to $N(P)$ at a vertex or on a side of $N(P)$. We put $N_{s}:=N(P) \cap$ $L_{s}$ and define

$$
\stackrel{\circ}{N}_{s}:=\left\{(j, \alpha) \in \mathbf{N}^{2} ; \alpha_{0 j \alpha}(0) \neq 0,(j+\alpha,-j) \in N_{s}\right\} .
$$

Now the principal part $P_{s}\left(D_{t}, D_{x}\right)$ and the Toeplitz symbol $f_{s}(z)$ associated with the Gevrey index $s$ are defined by,

$$
\begin{gathered}
P_{s}\left(D_{t}, D_{x}\right):=\sum_{(j, \alpha) \in \AA_{s}} a_{0 j \alpha}(0) D_{t}^{j} D_{x}^{\alpha}, \\
f_{s}(z):=\sum_{(j, \alpha) \in N_{s}} a_{0 j \alpha}(0) z^{-j} .
\end{gathered}
$$

We define Gevrey space $\mathscr{G}_{w}^{s}(R)(s, w, R>0)$ as follows.

Let $\mathbf{C}[[t, x]]$ denote the set of formal power series of variables $t, x \in \mathbf{C}$, and $\mathscr{O}(\Omega)$ the set of holomorphic functions on a domain $\Omega \subset \mathbf{C}_{t} \times \mathbf{C}_{x}$. Then the Gevrey space $\mathscr{G}_{w}^{s}(R)$ is defined by the following isomorphism of Frechét spaces (cf. Proposition 5.1 which follows),

$$
\mathrm{C}[[t, x]] \supset \mathscr{G}_{w}^{s}(R) \stackrel{\text { Borel transf. }}{\sim} \mathscr{O}\left(\left(\frac{|t|}{w}\right)^{1 / s}+|x|<R\right),
$$

where the Borel transformation is defined by

$$
\mathscr{G}_{w}^{s}(R) \ni \sum_{l, \beta \in \mathbf{N}} u_{l \beta} \frac{t^{l} x^{\beta}}{l ! \beta !} \mapsto \sum_{l, \beta \in \mathbf{N}} u_{l \beta} \frac{t^{l} x^{\beta}}{(s l) ! \beta !} \in \mathscr{O}\left(\left(\frac{|t|}{w}\right)^{1 / s}+|x|<R\right) .
$$

The factorial is defined by the gamma function, $r !:=\Gamma(r+1)$ for $r \geq 0$.

We consider the following Goursat problem in $\mathscr{G}_{w}^{s}(R)$,

$$
\left\{\begin{array}{l}
P\left(t, x ; D_{t}, D_{x}\right) u(t, x)=f(t, x) \in \mathscr{G}_{w}^{s}(R), \\
u(t, x)-v(t, x)=O\left(t^{j} x^{\alpha}\right), v(t, x) \in \mathscr{G}_{w}^{s}(R),
\end{array}\right.
$$


where $w(t, x)=O\left(t^{j} x^{\alpha}\right)$ in $\varphi_{w}^{s}(R)$ means that $w(t, x) t^{-j} x^{-\alpha} \in \mathscr{G}_{w}^{s}(R)$.

Now we can prove the following,

Theorem 0. Assume $\stackrel{\circ}{N}_{s} \neq \phi$ and $(j, \alpha) \in$ ch $\left\{\stackrel{\circ}{N}_{s}\right\}$, the convex hull of points in $\stackrel{\circ}{s}_{s}$. Further we assume

$$
f_{s}(z) \neq 0 \text { on }|z|=w \text {, and } \oint_{|z|=w} d\left(\log f_{s}(z) z^{\jmath}\right)=0 \text {. }
$$

Then the Goursat problem (0.7) has the Fredholm property for sufficiently small $R>0$. Precisely, there exists $R_{0}>0$ such that the mapping $P: t^{j} x^{\alpha} \mathscr{G}_{w}^{s}(R) \rightarrow \mathscr{G}_{w}^{s}(R)$ has the same finite dimensional kernel and cokernel for every $R$ such that $0<R<R_{0}$. Furthermore, if one of the following conditions is satisfied, then the problem (0.7) is uniquely solvable in $\mathscr{G}_{w}^{s}(R)$ for sufficiently small $R>0$ :

(i) $(j, \alpha)$ is an end point of $\operatorname{ch}\left\{\stackrel{\circ}{N}_{s}\right\}$.

(ii) There exists $c>0$ such that $\left\{f_{s}(z) z^{j} ;|z|=c\right\}$ is a segment.

(iii) There exists $c>0$ such that $0 \notin \operatorname{ch}\left\{f_{s}(z) z^{j} ;|z|=c\right\}$.

Moreover, every formal solution $u(t, x) \in \mathbf{C}[[t, x]]$ of the problem (0.7) (if it exists) belongs to $\mathscr{G}_{w}^{s}(R)$ for sufficiently small $R>0$.

We remark that if $s$ is an irrational, $\stackrel{\circ}{s}_{s}$ consists of an element whenever it is not empty, and the problem (0.7) is uniquely solvable in $\mathscr{G}_{w}^{s}(R)$ for every $w>0$ for sufficiently small $R>0([\mathrm{MH}])$. Therefore, our interest in this paper is the case where $s$ is a rational number and $\stackrel{\circ}{s}_{s}$ includes at least two elements. We also remark that the necessity of the condition $(0.8)$ will be made clear in Theorem 1 in Section 1.

The proof of Theorem 0, which will be completed in Section 5, is carried out by converting the problem to the Fredholm property of an integro-differential operator $L:=P D_{t}^{-j} D_{x}^{-\alpha}$ on Banach space $G_{w}^{s}(R)$ associated with $\mathscr{G}_{w}^{s}(R)$, which is defined in Section 1. Therefore, the main part of this paper is the study of Fredholm property of integro-differential operators on Banach spaces of Gevrey functions. The case of nonpositive Gevrey index will also be studied, but it is difficult to state a result for the Goursat problem in such a strict form as Theorem 0 .

At the end of this introduction, we give some historical background of the problem.

From the general theory of the Goursat problem, we can prove that the Goursat problem $(0.7)$ is uniquely solvable in $\mathscr{G}_{w}^{s}(R)$ for sufficiently small $R>0$ under the following so-called spectral condition, 


$$
\left|a_{0 j \alpha}(0)\right|>\sum_{(l, \beta) \in \mathcal{N}_{S} \backslash(j, \alpha)}\left|a_{0 l \beta}(0)\right| w^{j-l} .
$$

(See [H], [W], [M1], [M2] and [MH].)

On the other hand, some attempts to make analysis of interior points of spectral radius were made by many authors for special operators after the work by Leray ([G], [L], [Ys1], [Ys2]). Leray studied the following Goursat problem in the category of local holomorphic functions at the origin.

$$
\left(\lambda D_{t} D_{x}-D_{t}^{2}-D_{x}^{2}\right) u(t, x)=f(t, x), u(t, x)=O(t x) .
$$

He proved that the problem is uniquely solvable if $\lambda \in \mathbf{C} \backslash[-2,2]$. He also studied the case where $\lambda \in[-2,2]$, and made clear that the solvability of the problem depends deeply on the diophantine nature of the number $\lambda$. In [M3], Miyake proved that the interval $[-2,2]$ is a spectral set of an associated integrodifferential operator with the Goursat problem (see Theorem 1 and Example 1.1 in Section 1). Yoshino made a series of studies in this direction in the category of local holomorphic functions ([Ys1,2,3,4,5]). Leray's example was extended by Miyake [M3] in Gevrey spaces of positive index and also of non positive index. A prototype of results in this paper was given there, but a condition like (0.8) was not awared, which enables us to study general operators.

In Section 1, we shall state our results on Fredholm properties for integrodifferential operators on Banach space of Gevrey functions after some preparations. In Section 2, we shall study invertibility or norm inequalities for (finite section) Toeplitz matrices on (finite section) weighted $l^{p}$ space $(1 \leq p \leq \infty)$, where we shall see that how the condition $(0.8)$ does play a crucial role in our study. In Sections 3 and 4 , we shall give the proofs of the results, and Theorem 0 will be proved in Section 5 .

\section{Statement of results}

Let $L \equiv L\left(t, x ; D_{t}, D_{x}\right)$ be an integro-differential operator of finite order with holomorphic coefficients in a neighbourhood of the origin of $\mathbf{C}_{t} \times \mathbf{C}_{x}$, and write it in the form,

$$
L=\sum_{\sigma \in \mathbf{N}} \sum_{j, \alpha \in \mathbf{Z}}^{\text {finite }} a_{\sigma j \alpha}(x) t^{\sigma} D_{t}^{j} D_{x}^{\alpha},
$$

where $\mathbf{Z}$ denotes the set of integers. The symbol $D_{t}^{-1}$ denotes an integration from 0 to $t$ in the variable $t$ in the formal sense. It is the same for $D_{x}^{-1}$.

The Newton polygon $N(L)$ of the operator $L$ is defined in the same manner as 
(0.2) for partial differential operators by replacing $\mathbf{N}$ by $\mathbf{Z}$.

We assume that the Newton polygon $N(L)$ has a side $N_{s}$ with slope $k=$ $1 /(s-1)(s \in \mathbf{Q})$ which contains the origin.
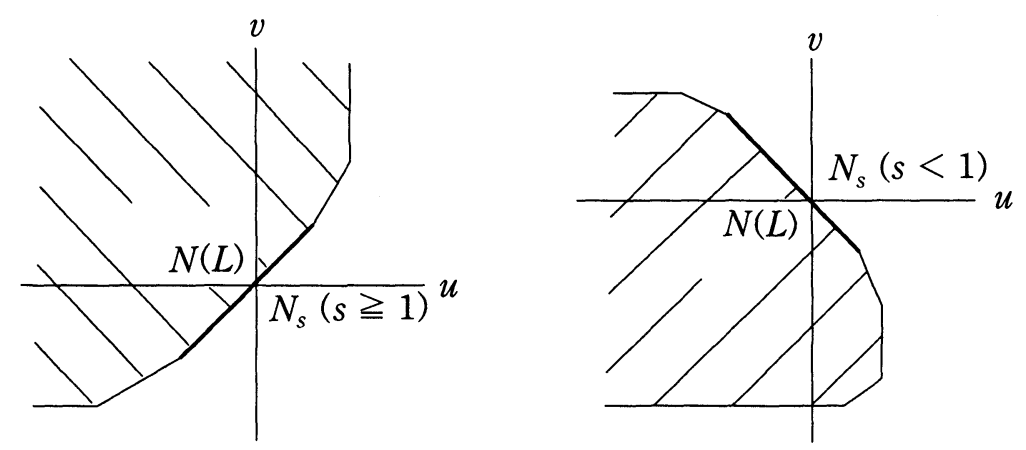

This assumption implies that

$$
\begin{aligned}
& s j+(1-s) \sigma+\alpha \leq 0 \text { if } a_{\sigma j \alpha}(x) \not \equiv 0, \\
& s j+(1-s) \sigma+\alpha=0\left(a_{\sigma j \alpha}(x) \not \equiv 0\right) \text { if and only if }(j+\alpha, \sigma-j) \in N_{s} .
\end{aligned}
$$

In the case $s<1$ it is assumed, a priori, that the operator has polynomial coefficients in the variable $t$. As a fundamental assumption, we impose the following condition throughout this paper.

$$
\stackrel{\circ}{N}_{s}:=\left\{(j, \alpha) \in \mathbf{Z} \times \mathbf{Z} ; a_{0 j \alpha}(0) \neq 0,(j+\alpha,-j) \in N_{s}\right\} \neq \phi .
$$

We decompose the operator $L$ as follows.

$$
L=L_{0}\left(D_{t}, D_{x}\right)+L_{1}\left(t, x ; D_{t}, D_{x}\right)+L_{2}\left(t, x ; D_{t}, D_{x}\right)
$$

where

$$
\begin{aligned}
& L_{0}=\sum_{(j, \alpha) \in N_{s}} a_{0 j \alpha}(0) D_{t}^{j} D_{x}^{\alpha} \quad \text { (the principal part), } \\
& L_{1}=\sum_{s j+(1-s) \sigma+\alpha=0} a_{\sigma j \alpha}(x) t^{\sigma} D_{t}^{j} D_{x}^{\alpha}, \quad a_{0 j \alpha}(0)=0 \text { or } \sigma \geq 1, \\
& L_{2}=\sum_{s j+(1-s) \sigma+\alpha<0}^{\text {finite }} a_{\sigma j \alpha}(t, x) t^{\sigma} D_{t}^{j} D_{x}^{\alpha} .
\end{aligned}
$$

We shall study the Fredholm property and the bijectivity of the following mappings on Banach spaces, 


$$
\begin{aligned}
& L: G_{w}^{s}(R) \rightarrow G_{w}^{s}(R)(s, w, R>0), \\
& L: G_{w}^{s}(R ; n) \rightarrow G_{w}^{s}(R ; n)(s \leq 0, w, R>0, n \in \mathbf{N}) .
\end{aligned}
$$

Throughout this paper, a bounded operator $L$ on a Banach space $X$ is said to be a Fredholm operator if it is a Fredholm operator with an index 0 , that is, $L$ has the same finite dimensional kernel and cokernel.

We define Banach spaces $G_{w}^{s}(R)$ and $G_{w}^{s}(R ; n)$ as follows.

Definition. Let $U(t, x)=\sum_{l, \beta \in \mathbf{N}} U_{l \beta} t^{l} x^{\beta} / l ! \beta ! \in \mathbf{C}[[t, x]]$. Then we define:

(i) $U(t, x) \in G_{w}^{s}(R)(s>0) \stackrel{\text { def }}{\Longleftrightarrow}$

$$
\|U\|_{w, R}^{(s)}:=\sum_{l, \beta \in \mathbf{N}}\left|U_{l \beta}\right| \frac{w^{l} R^{s l+\beta}}{(s l+\beta) !}<\infty .
$$

(ii) $U(t, x) \in G_{w}^{s}(R ; n)(s \leq 0) \stackrel{\text { def }}{\Longleftrightarrow}$

$$
\|U\|_{w, R, n}^{(s)}:=\inf \left\{C ;\left|U_{l \beta}\right| \leq C \frac{1}{w^{l} R^{s l+\beta}} \frac{(n l+(n+1) \beta) !}{((n-s) l+n \beta) !}\right\}<\infty .
$$

The Toeplitz symbol $f(z)$ of the operator $L$ (associated with the above mapping) is defined by

$$
f(z):=\sum_{(j, \alpha) \in \mathfrak{N}_{s}} a_{0 j \alpha}(0) z^{-j} \in \mathbf{C}\left[z, z^{-1}\right]
$$

where $\mathbf{C}\left[z, z^{-1}\right]$ denotes the set of polynomials of $z$ and $z^{-1}$.

When $f(z) \neq 0$ on a circle $K_{w}:=\{z \in \mathbf{C} ;|z|=w\}$, we denote by $I_{w}(f)$ the winding number of $f(z)$ at the origin with respect to the circle $K_{w}$,

$$
I_{w}(f):=\frac{1}{2 \pi i} \oint_{|z|=w} d(\log f(z)) .
$$

Now our results are stated as follows.

THEOREM 1 (The case $s>0$ ). (i) Suppose the following condition, $(\mathrm{H})_{w}$

$$
f(z) \neq 0 \text { on } K_{w} \text {, and } I_{w}(f)=0 .
$$

Then there exists a positive constant $R_{0}$ such that $L$ is a Fredholm operator on $G_{w}^{s}(R)$ for every $0<R \leq R_{0}$. Furthermore if we define an ideal $M^{s}[N]$ of $\mathbf{C}[[t, x]]$ by

$$
\mathcal{M}^{s}[N]:=\left\{U(t, x) \in \mathbf{C}[[t, x]] ; U_{l \beta}=0 \text { for } \quad s l+\beta<N\right\},
$$


and $G_{w}^{s}(R)[N]:=G_{w}^{s}(R) \cap \mathcal{M}^{s}[N]$, then $L$ is bijective on $\mathcal{M}^{s}[N]$ and also on $G_{w}^{s}(R)[N]$ for sufficiently large $N$ and small $R>0$. Therefore, $L$ is bijective on $\mathbf{C}[[t, x]] / G_{w}^{s}(R)$ for sufficiently small $R>0$.

(ii) Suppose $L_{1}=0$ in the decomposition (1.3). Then $L$ is a Fredholm operator on $G_{w}^{s}(R)$ for some $R>0$ if and only if the condition $(\mathrm{H})_{w}$ is satisfied. Furthermore, the resolvent set of the operator $L_{0}$ on $G_{w}^{s}(R)$ is invariant on $R>0$, which we denote by $\rho\left(L_{0}\right)$, and we have

$$
\rho\left(L_{0}\right)=\underset{R>0}{\cup} \rho\left(L ; G_{w}^{s}(R)\right),
$$

where $\rho\left(L ; G_{w}^{s}(R)\right)$ denotes the resolvent set of the operator $L$ on $G_{w}^{s}(R)$.

Remark 1.1. If $s \geq 1$ in the above theorem, we may assume that the coefficients of the operator belong to $\cup_{R>0} G_{w}^{s}(R)$ (cf. Lemma 4.2).

Remark 1.2. In course of the proof, we will see that $\lambda \in \mathbf{C}$ belongs to the resolvent set of $L$ on $G_{w}^{s}(R)$ (for sufficiently small $R>0$ ) if one of the following conditions is satisfied:

(i) $f(z)$ is a polynomial of $z$ or $z^{-1}$ and $(\mathrm{H})_{w}$ is satisfied for $f(z)-\lambda$.

(ii) $\left\{f(z) ; z \in K_{c}\right\}$ is a segment for some $c>0$ and $(\mathrm{H})_{w}$ is satisfied for $f(z)-\lambda$. In this case, by Szegö's theorem stated in Section 2, $\sigma_{p}\left(L_{0}\right)$, the set of eigenvalues of $L_{0}$ on $G_{w}^{s}(R)$, is invariant for $w>0$ and is densely distributed on this segment.

(iii) $\lambda \notin \operatorname{ch}\left\{f(z) ; z \in K_{c}\right\}$ for some $c>0$ and $(\mathrm{H})_{w}$ is satisfied for $f(z)-\lambda$. Furthermore the set of eigenvalues, which is included in a domain $\left\{\lambda \in \mathbf{C} ;(\mathrm{H})_{w}\right.$ is satisfied for $f(z)-\lambda$, consists of finite points.

THEOREM 2 (The case $s \leq 0$ ). Let assume $(\mathrm{H})_{c w}$ is satisfied for $c=e^{s-1}$ and $c=e^{s(s-1)}$. Then there exist $R_{0}>0$ and $n_{0} \in \mathbf{N}$ such that $L$ is a bijection on $G_{w}^{s}(R ; n)$ for $0<R \leq R_{0}$ and $n \geq n_{0}$. Precisely, it holds that

$$
\begin{aligned}
& \cup \underset{R>0}{\cup} \underset{n \in \mathbf{N}}{\cup} \rho\left(L ; G_{w}^{s}(R ; n)\right) \\
& \quad \supset\left\{\lambda \in \mathbf{C} ; f(z)-\lambda \text { satisfies }(\mathrm{H})_{c w} \text { for } c=e^{s-1} \text { and } c=e^{s(s-1)}\right\},
\end{aligned}
$$

where $\rho\left(L ; G_{w}^{s}(R ; n)\right)$ denotes the resolvent set of $L$ on $G_{w}^{s}(R ; n)$.

Our theorems are proved first by showing the same results for the principal part $L_{0}$ in Section 3 , and then by applying the stability of Fredholm property by 
small perturbations in Section 4. The results for $L_{0}$ will be proved by showing that an equation $L_{0} U(t, x)=F(t, x)$ in $G_{w}^{s}(R)$ (resp. in $G_{w}^{s}(R ; n)$ ) is decomposed into an infinite direct product of finite section Wiener-Hopf equations on finite section spaces of weighted $l^{1}$ space (resp. Wiener-Hopf equations on weighted $l^{\infty}$ spaces). In these studies, we shall see that the Fredholm property depends deeply on the invertibility or the norm inequality for (finite section) Toeplitz matrices which holds under the condition $(\mathrm{H})_{w}$ (cf. Propositions $2.2,2.3$ ).

We give examples below.

EXAmple 1.1 ([M3]). Let $L_{0}=D_{t}^{p} D_{x}^{-p-\alpha}+D_{t}^{-p} D_{x}^{p+\alpha}(p \geq 1, p+\alpha>0)$. The Gevrey index of this operator is $s=1+(\alpha / p)>0$, and the Toeplitz symbol is $f(z)=z^{-p}+z^{p}$. Since $\{f(z) ;|z|=1\}=[-2,2]$, by Remark 1.2 (ii) or (iii), we have

$$
\begin{aligned}
\rho\left(L_{0}\right) & =\mathbf{C} \backslash \operatorname{ch}\left\{f(z) ; z \in K_{w}\right\} \\
& =\left\{\lambda \in \mathbf{C} ;\left(\frac{\operatorname{Re} \lambda}{w^{p}+w^{-p}}\right)^{2}+\left(\frac{\operatorname{Im} \lambda}{w^{p}-w^{-p}}\right)^{2}>1\right\}(\subset \mathbf{C} \backslash[-2,2]),
\end{aligned}
$$

and $\sigma_{p}\left(L_{0}\right)$ is densely distributed on $[-2,2]$. More precisely, we have

$$
\sigma_{p}\left(L_{0}\right)=\bigcup_{n=0}^{\infty}\{2 \cos \pi \theta ; \sin (n+2) \pi \theta=0,0<\theta<1\},
$$

from results in [M3], where the case $w=1$ was studied.

Example 1.2. Let $L_{0}=\frac{4}{3} D_{t}^{-2} D_{x}^{2+2 \alpha}+3 D_{t}^{-1} D_{x}^{1+\alpha}-\frac{9}{4} D_{t} D_{x}^{-1-\alpha}$, where $\alpha \geq 1$. Then the Gevrey index of this operator is given by $s=1+\alpha \geq 2$ and the Toeplitz symbol is given by $f(z)=\frac{4}{3} z^{2}+3 z-\frac{9}{4} z^{-1}=\left(\frac{4}{3}-z^{-1}\right)\left(z+\frac{3}{2}\right)^{2}$. Therefore the condition $(\mathrm{H})_{w}$ is satisfied for $\frac{3}{4}<w<\frac{3}{2}$. Then the operator $L_{0}$ is a Fredholm operator on $G_{w}^{s}(R)$ for $\frac{3}{4}<w<\frac{3}{2}$. In this case, it holds that $0 \in$ $\cap_{3 / 4<w<3 / 2} \operatorname{ch}\{f(z) ;|z|=w\}$ (see the figures). From the proof of Theorem 1 we can see that $L_{0}$ has $1+\alpha$ dimensional kernel and cokernel on $G_{w}^{s}(R)$. (Cf. Example 2.1.) 


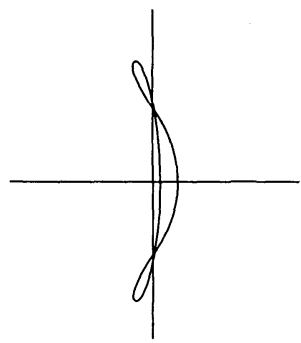

$w=0.8$

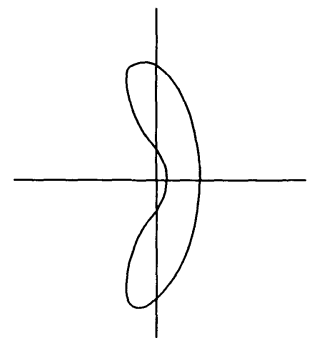

$w=1$

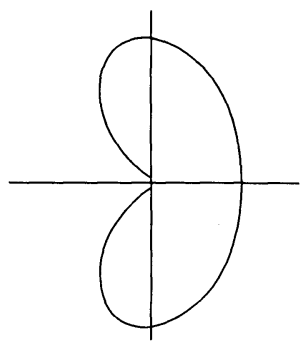

$w=1.3$

\section{Inversion and norm inequality of Toeplitz matrices}

We define Banach spaces $l_{p, w}(p=1$ or $\infty, w>0)$ of formal Laurent series by

$$
\begin{aligned}
& l_{1, w}:=\left\{u(z)=\sum_{j \in \mathbf{Z}} u_{j} z^{j} ;\|u\|_{1, w}:=\sum_{j \in \mathbf{Z}}\left|u_{j}\right| w^{j}<\infty\right\}, \\
& l_{\infty, w}:=\left\{u(z)=\sum_{j \in \mathbf{Z}} u_{j} z^{j} ;\|u\|_{\infty, w}:=\sup \left\{\left|u_{j}\right| w^{j} ; j \in \mathbf{Z}\right\}<\infty\right\} .
\end{aligned}
$$

We, sometimes, identify $u(z) \in l_{p, w}$ with a sequence $u=\left\{u_{j}\right\}_{j \in \mathbf{Z}}$ with the above defined norm. We denote by $l_{p, w}^{+}$(resp. $l_{p, w}^{-}$) the set of $u(z) \in l_{p, w}$ with $u_{j}=0$ for $j<0$ (resp. $j>0$ ). The projection $P: l_{p, w} \rightarrow l_{p, w}^{+}$is naturally defined by

$$
P(u)=\sum_{j \in \mathbf{N}} u_{j} z^{j} \quad \text { for } \quad u(z)=\sum_{j \in \mathbf{Z}} u_{j} z^{j} \in l_{p, w} .
$$

For $f(z)=\sum_{j=-m}^{n} f_{j} z^{j} \in \mathbf{C}\left[z, z^{-1}\right]\left(-m<n, f_{-m} f_{n} \neq 0\right)$, a Wiener-Hopf equation on $l_{p, w}^{+}$with symbol $f(z)$ is defined by

$$
P_{f}[u]:=P(f u)=g(z) \in l_{p, w}^{+}\left(u(z) \in l_{p, w}^{+}\right) .
$$

The operator $P_{f}$ is called a Toeplitz operator with symbol $f(z)$. An infinite matrix $T_{f}$ defined by

$$
T_{f}:=\left(f_{j-k}\right)_{j, k=0,1,2, \ldots}
$$

is said to be a Toeplitz matrix with symbol $f(z)$. Then the equation (2.3) is written in the matrix form by

$$
T_{f} u=g \in l_{p, w}^{+} \quad\left(u \in l_{p, w}^{+}\right) .
$$

We note that $P_{f}$ or $T_{f}$ defines a bounded operator on $l_{p, w}^{+}$with operator norm,

$$
\left\|P_{f}\right\|=\left\|T_{f}\right\|=\|f\|_{1, w} .
$$


The first purpose of this section is to study the unique solvability of the Wiener-Hopf equation (2.3) or (2.3) on $l_{p, w}^{+}$. For that purpose, we firstly investigate the condition $(\mathrm{H})_{w}$. The condition $(\mathrm{H})_{w}$ is satisfied if and only if $n, m \geq 0$ and the equation $z^{m} f(z)=0$ has $m$ roots $\left\{\mu_{j}\right\}_{j=1}^{m}$ in $\{0<|z|<w\}$ and $n$ roots $\left\{\mu_{m+j}\right\}_{j=1}^{n}$ in $\{|z|>w\}$. Therefore, under the condition $(\mathrm{H})_{w}$, we have a decomposition, $f(z)=f_{+}(z) f_{-}(z)$, given by

$$
f_{+}(z)=\prod_{j=1}^{n}\left(z-\mu_{m+j}\right), \quad f_{-}(z)=\prod_{j=1}^{m}\left(1-\frac{\mu_{j}}{z}\right),
$$

where we assumed $f_{n}=1$ without loss of generality. Note that $f_{+}(z)$ (resp. $f_{-}(z)$ ) is holomorphic and does not vanish on $\{|z| \leq w\}$ (resp. $\{w \leq|z| \leq \infty\}$ ). Such a decomposition is called a Hilbert factorization of $f(z)$ with respect to the circle $K_{w}$. We note that the converse holds, that is, $f(z)$ is Hilbert factorizable with respect to the circle $K_{w}$ if and only if $f(z)$ satisfies the condition $(\mathrm{H})_{w}$. We omit the proof, since it is easy.

From the decomposition, we see that

$$
f_{ \pm}(z)^{-1}=\sum_{j \in \mathbf{N}} b_{ \pm j} z^{ \pm j}
$$

are absolutely convergent on the circle $K_{w}$, and hence they belong to $l_{1, w}$. These facts imply that Toeplitz matrices $T_{f_{ \pm}^{-1}}$ define bounded operators on $l_{p, w}^{+}$with operator norms

$$
\left\|T_{f_{ \pm}^{-1}}\right\|=\left\|f_{ \pm}(z)^{-1}\right\|_{1, w}\left(\equiv \sum_{j \in \mathbf{N}}\left|b_{ \pm j}\right| w^{ \pm j}\right) .
$$

Under the above consideration, we see that the condition $(\mathrm{H})_{w}$ implies the following decompositions of Toeplitz operator $P_{f}$ and Toeplitz matrix $T_{f}$,

$$
P_{f}=P_{f-} P_{f+} \text { and } T_{f}=T_{f-} T_{f+},
$$

where $P_{f+}$ is a multiplier operator by $f_{+}(z)$. Note that $T_{f+}$ (resp. $T_{f_{-}}$) is a lower (resp. an upper) triangle matrix, and has a bounded inverse matrix on $l_{p, w}^{+}$given by $T_{f+}^{-1}=T_{f_{+}^{-1}}$ (resp. $T_{f-}^{-1}=T_{f_{-}^{-1}}$ ). Thus we have seen that under the condition $(\mathrm{H})_{w}, T_{f}$ has a bounded inverse matrix on $l_{p, w}^{+}$given in the form, $T_{f}^{-1}=T_{f_{+}^{-1}} T_{f_{-}^{-1}}$, and its operator norm is estimated by

$$
\left\|T_{f}^{-1}\right\| \leq\left\|f_{+}(z)^{-1}\right\|_{1, w}\left\|f_{-}(z)^{-1}\right\|_{1, w} .
$$

This estimate is obtained from the following Hausdorff-Young inequality of discrete type. 
Lemma 2.1. Let $a(z) \in l_{1, w}$ and $b(z) \in l_{p, w}$, where $1 \leq p \leq \infty$. Then $a(z) b(z) \in l_{p, w}$ and we have $\|a b\|_{p, w} \leq\|a\|_{1, w}\|b\|_{p, w}$. Here the Banach space $l_{p, w}$ $(1<p<\infty)$ is defined in the similar manner as in the case $p=1$.

We omit the proof, since it is obvious. It is important that the converse holds.

Proposition 2.2. The Toeplitz matrix $T_{f}$ is bijective, that is, invertible on $l_{p, w}^{+}$if and only if the condition $(\mathrm{H})_{w}$ is satisfied. Moreover, suppose that $f(z) \neq 0$ on $K_{w}$ and

$$
I_{w}(f):=\frac{1}{2 \pi i} \oint_{|z|=w} d(\log f(z))=k \neq 0 .
$$

If $k>0$ then $T_{f}$ is injective with $k$ dimensional cokernel, and if $k<0$ then $T_{f}$ is surjective with $-k$ dimensional kernel.

Remark 2.1. Calderón, Spitzer and Widom ([CSW]) proved this result in the case where $p=\infty$ and $w=1$. The above consideration and the proof below show that this proposition holds for every $1 \leq p \leq \infty$.

Proof. We may assume $w=1$ without loss of generality. Indeed, it is sufficient to make a change $z$ by $w z$. This change implies,

$$
\begin{aligned}
& l_{p, w}^{+} \ni\left\{u_{j}\right\}_{j \in \mathbf{N}} \tilde{\mapsto}\left\{u_{j} w^{j}\right\}_{j \in \mathbf{N}} \in l_{p, 1}^{+}, \\
& T_{f} \text { on } l_{p, w}^{+} \mapsto D_{\infty}(w) T_{f} D_{\infty}\left(w^{-1}\right) \text { on } l_{p, 1}^{+},
\end{aligned}
$$

where $D_{\infty}(w):=\operatorname{diag}\left\{1, w, \ldots, w^{j}, \ldots\right\}$, a diagonal matrix with the $j$-th diagonal component $w^{j}(j \geq 0)$, and $D_{\infty}^{-1}(w)=D_{\infty}\left(w^{-1}\right)$.

We prove the "only if" part, so we always assume that $T_{f}$ is bijective on $l_{p}^{+}$. In what follows, we omit the suffix $w=1$, and the condition $(\mathrm{H})_{w}$ is denoted by (H). We give the proof dividing into two parts. The proof of latter half of the statement will be given in part 2 .

1) The case $f(z) \in \mathbf{C}[z]$ or $\mathbf{C}\left[z^{-1}\right]$.

First, we consider the case $f(z)=\sum_{j=0}^{n} f_{j} z^{j} \in \mathbf{C}[z](n>0)$. We put $f(z)=$ $z^{k} g(z), g(0) \neq 0, k \geq 0$. Then the Toeplitz matrix $T_{f}$ is decomposed into

$$
T_{f}=S_{+}(k) T_{g}=T_{g} S_{+}(k),
$$

where $S_{+}(k)$ is a shift operator defined by

$$
S_{+}(k):{ }^{t}\left(u_{0}, u_{1}, u_{2}, \ldots\right) \mapsto{ }^{t} \overbrace{(0, \ldots, 0}^{k}, u_{0}, u_{1}, u_{2}, \ldots) .
$$


This shows that if $k>0$, the cokernel of $T_{f}$ includes $k$ dimensional vector space, and hence $T_{f}$ is not surjective. Now we consider the case where $f(0) \neq 0$. Suppose that the condition $(\mathrm{H})$ is not satisfied, that is, $f(z)=0$ has at least one root in $\{|z| \leq 1\}$.

First assume that $f(z)=0$ has at least one root in $\{|z|<1\}$. This implies that the convergent radius of $f(z)^{-1}:=\sum_{j \geq 0} b_{j} z^{j}$ is less than 1 . Hence we have

$$
\limsup _{j \rightarrow \infty}\left|b_{j}\right|=\infty \text {. }
$$

We put $e_{j}={ }^{t}(0, \ldots, 0, \stackrel{j}{1}, 0, \ldots)(j \geq 0)$. Then $e_{j}$ does dot belong to the image of $T_{f}$. Indeed, the equation, $T_{f} u=e_{j}$, has a unique solution,

$$
u_{j}={ }^{t} \overbrace{(0, \ldots, 0}^{j}, b_{0}, b_{1}, b_{2}, \ldots),
$$

which satisfies the equation in the formal sense, but does not belong to $l_{p}^{+}$.

Next suppose that $f(z)=0$ has all roots in $\{|z| \geq 1\}$ and has at least one root on $\{|z|=1\}$. It is assumed that $T_{f}$ is invertible on $l_{p}^{+}$. Since the set of invertible operators on $l_{p}^{+}$consists of an open set in the set of bounded operators, there exists a positive constant $\varepsilon>0$ such that every bounded operator $B$ on $l_{p}^{+}$ satisfying $\left\|T_{f}-B\right\|<\varepsilon$ is also bijective on $l_{p}^{+}$, where $\|\cdot\|$ denotes the operator norm. For any $\varepsilon>0$, we can give a polynomial $g(z) \in \mathbf{C}[z]$ by a small change of coefficients of $f(z)$ such that $g(z)=0$ has at least one root in $\{|z|<1\}$, and also $\left\|T_{f}-T_{g}\right\|<\varepsilon$. This implies that $T_{f}$ is not bijective on $l_{p}^{+}$.

Next, we consider the case $f(z)=\sum_{j=-m}^{0} f_{j} z^{j} \in \mathbf{C}\left[z^{-1}\right](m>0)$. We put $f(z)=z^{-k} g(z), k \geq 0$ and $g(\infty) \neq 0$. Then $T_{f}$ has the following decomposition,

$$
T_{f}=S_{-}(k) T_{g}=T_{g} S_{-}(k),
$$

where $S_{-}(k)$ is a shift operator defined by

$$
S_{-}(k):{ }^{t}\left(u_{0}, u_{1}, u_{2}, \ldots\right) \mapsto{ }^{t}\left(u_{k}, u_{k+1}, u_{k+2}, \ldots\right) .
$$

Therefore if $k>0$ then operator $T_{f}$ includes $k$ dimensional kernel, and hence it is not injective. So we may assume that $f(\infty) \neq 0$. Suppose that $f(z)$ does not satisfy the condition $(\mathrm{H})$, that is, $f(z)=0$ has at least one root in $\{|z| \geq 1\}$. By the same reason as above, we only consider the case that $f(z)=0$ has at least one root in $\{|z|>1\}$ to prove that $T_{f}$ is not bijective on $l_{p}^{+}$. We put $f(z)^{-1}:=\sum_{j=0}^{\infty}$ $b_{-j} z^{-j}$. Then we have

$$
\limsup _{j \rightarrow \infty}\left|b_{-j}\right|=\infty
$$

It is easy to check 


$$
T_{f} T_{f^{-1}} e_{j}=e_{j}(j=0,1,2, \ldots), \quad T_{f^{-1}} e_{j}={ }^{t}\left(b_{-j}, b_{-j+1}, \ldots, b_{0}, 0, \ldots\right) .
$$

This shows that $T_{f}$ does not have bounded inverse on $l_{p}^{+}$, because

$$
\left\|T_{f^{-1}} e_{j}\right\|_{p} \rightarrow \infty \quad \text { as } j \rightarrow \infty .
$$

2) The case $f(z)=\sum_{j=-m}^{n} f_{j} z^{j}\left(m, n>0, f_{-m} f_{n} \neq 0\right)$.

Let $\left\{\mu_{j}\right\}_{j=1}^{m+n}$ be the roots of an equation $z^{m} f(z)=0$, and suppose that

$$
\left|\mu_{j}\right|<1(1 \leq j \leq p), \quad\left|\mu_{p+j}\right|=1(1 \leq j \leq q),\left|\mu_{p+q+j}\right|>1(1 \leq j \leq r),
$$

where $p, q, r \geq 0$ and $p+q+r=m+n$.

In what follows, we assume that the condition $(\mathrm{H})$ is not satisfied.

First, we consider the case $q=0$. Hence it holds that $p<m$ or $p>m$. We consider the following decomposition,

$$
f(z)=z^{p-m} \prod_{j=1}^{p}\left(1-\frac{\mu_{j}}{z}\right) \prod_{j=1}^{r}\left(z-\mu_{p+j}\right) \stackrel{\text { put }}{=} z^{p-m} f_{-}(z) f_{+}(z)
$$

According to the case $p<m$ or $p>m$, we have the following decompositions of the Toeplitz matrix $T_{f}$,

$$
T_{f}=S_{-}(m-p) T_{f_{-}} T_{f_{+}}(p<m), \quad T_{f}=T_{f_{-}} T_{f_{+}} S_{+}(p-m)(p>m) .
$$

Since $T_{f_{ \pm}}$are bijective on $l_{p}^{+}$, we have $T_{f}$ has $(m-p)$ dimensional kernel or $(p-m)$ dimensional cokernel according to the case $p<m$ or $p>m$. This proves the latter half of the statement in the proposition. Indeed, the number $I(f)$ defined by (2.7) for $w=1$ is given by $I(f)=p-m$ in this case.

Next, we consider the case $q \neq 0$.

First, we assume $p \geq m$. Then we have the following decomposition,

$$
f(z)=z^{p-m} \prod_{j=1}^{p}\left(1-\frac{\mu_{j}}{z}\right) \prod_{j=1}^{q+r}\left(z-\mu_{p+j}\right) \stackrel{\text { put }}{=} z^{p-m} f_{-}(z) f_{+}(z) .
$$

This implies the following decomposition of $T_{f}$,

$$
T_{f}=T_{f_{-}} T_{f_{+}} S_{+}(p-m) .
$$

Since $T_{f_{-}}$is bijective on $l_{p}^{+}, T_{f_{+}} S_{+}(p-m)$ should be bijective on $l_{p}^{+}$. But this is impossible, because if $p>m$ then this operator has positive dimensional cokernel, and also if $p=m$ then $T_{f_{+}}$is not bijective from the fact that $q>0$ as we have proved in the first part.

Next, we assume $p+q \leq m$. Then we have the following decomposition, 


$$
f(z)=z^{p+q-m} \prod_{j=1}^{p+q}\left(1-\frac{\mu_{j}}{z}\right) \prod_{j=1}^{r}\left(z-\mu_{p+q+j}\right) \stackrel{\text { put }}{=} z^{p+q-m} f_{-}(z) f_{+}(z)
$$

This implies the following decomposition of $T_{f}$,

$$
T_{f}=S_{-}(m-p-q) T_{f_{-}} T_{f_{+}} .
$$

Since $T_{f_{+}}$is bijective on $l_{p}^{+}, S_{-}(m-p-q) T_{f_{-}}$should be bijective on $l_{p}^{+}$. But this is impossible, because if $p+q<m$ then this operator has a positive dimensional kernel, and also if $p+q=m$ then $T_{f_{-}}$is not bijective as we have proved in the first part.

The above considerations show that it should hold that $p<m, p+q>m$ and $r<n$ whenever $T_{f}$ is bijective on $l_{p}^{+}$. On the other hand, by a small perturbation of the coefficients of $f(z)$, we get $g(z)$ such that $g(z)=0$ has $p+q$ roots in $\{|z|<1\}$ and $g(z) \neq 0$ on $\{|z|=1\}$. Then $T_{g}$ is not bijective on $l_{p}^{+}$. This, combining with the reasoning in the first part, implies that $T_{f}$ is not bijective on $l_{p}^{+}$if the condition $(\mathrm{H})$ is not satisfied.

Thus the proof is completed.

Next, we study the solvability of finite section Wiener-Hopf equation.

Let $u^{(N)}(z)=\sum_{j=0}^{N} u_{j} z^{j}$ and $g^{(N)}(z)=\sum_{j=0}^{N} g_{j} z^{\prime}$ for $N \in \mathbf{N}$. Then an $N$-th finite section Wiener-Hopf equation with symbol $f(z)$ is defined by

$$
P_{f}\left[u^{(N)}\right]-g^{(N)}(z)=O\left(z^{N+1}\right),
$$

where $O\left(z^{N+1}\right)$ denotes the formal power series with power larger than $N$. An $N$-th finite section Toeplitz matrix $T_{f}(N)$ with symbol $f(z)=\sum f_{j} z^{j} \in \mathbf{C}\left[z, z^{-1}\right]$ is defined by

$$
T_{f}(N):=\left(f_{j-k}\right)_{j, k=0,1,2, \ldots, N} .
$$

Then the equation (2.8) is written in the matrix form by

$$
T_{f}(N) u^{(N)}=g^{(N)} \in \mathrm{C}^{N+1},
$$

where $u^{(N)}:={ }^{t}\left(u_{0}, u_{1}, \ldots, u_{N}\right)$ and so on. We take an induced norm $\left\|u^{(N)}\right\|_{1, w ; N}$ from $l_{1, w}^{+}$, that is,

$$
\left\|u^{(N)}\right\|_{1, w ; N}:=\sum_{j=0}^{N}\left|u_{j}\right| w^{j} .
$$

We denote by $l_{1, w}[N]$ the space $\mathbf{C}^{N+1}$ equipped with this norm. Now we can prove the following proposition corresponding to Proposition 2.2 . 
Proposition 2.3. Let $f(z)$ satisfy the condition $(\mathrm{H})_{w}$. Then there exists $N_{0} \in \mathbf{N}$ such that the equation (2.8) is uniquely solvable for every $N \geq N_{0}$ and the following norm inequality holds.

$$
\left\|u^{(N)}\right\|_{1, w ; N} \leq K\left\|g^{(N)}\right\|_{1, w ; N}\left(N \geq N_{0}\right),
$$

where the constant $K$ is independent of $N\left(\geq N_{0}\right)$. Conversely, the norm inequality (2.9) implies the condition $(\mathrm{H})_{w}$. Hence the norm inequality (2.9) holds if and only if the condition $(\mathrm{H})_{w}$ holds.

Remark 2.2. Let $\left\|u^{(N)}\right\|_{p, w ; N}$ be the induced norm from $l_{p, w}^{+}(1 \leq p \leq \infty)$. Then the proof below shows that the condition $(\mathrm{H})_{w}$ implies the norm inequality (2.9) for every $1 \leq p \leq \infty$. However, we can conclude that the norm inequality (2.9) implies the condition $(\mathrm{H})_{w}$ if $1 \leq p<\infty$, but we do not know whether it does hold in the case $p=\infty$.

Proof. We assume $w=1$ in what follows, and the condition $(\mathrm{H})_{w}$ is only denoted by $(\mathrm{H})$ as pointed out at the beginning of the proof of Proposition 2.2. We denote by $l_{1}[N]$ the space $\mathbf{C}^{N+1}$ equipped with the induced norm from $l_{1,1}^{+}$, and the norm is denoted by $\left\|u^{(N)}\right\|_{1 ; N}$. The norm of $u \in l_{1}$ (or $l_{1}^{ \pm}$) is denoted by $\|u\|_{1}$.

For the proof of the former half of the statement, it is sufficient to prove the norm inequality (2.9) for sufficiently large $N$. Indeed, (2.9) implies the uniqueness of solutions, and the solvability follows from the uniqueness of solutions for linear equations of determined type. We follow the argument by Baxter [B]. The equation (2.8) is equivalent to

$$
f(z) u^{(N)}(z)=H_{-}(z)+g^{(N)}(z)+H_{+}(z),
$$

for some $H_{ \pm}(z) \in l_{1}^{ \pm}$of the form,

$$
H_{-}(z)=\sum_{j=-\infty}^{-1} h_{j} z^{j}, \quad H_{+}(z)=\sum_{j=N+1}^{\infty} h_{j} z^{j}
$$

It is sufficient to prove the following inequalities,

$$
\left\|H_{-} f_{-}^{-1}\right\|_{1} \leq C\left\|g^{(N)}\right\|_{1 ; N}, \quad\left\|H_{+} f_{+}^{-1}\right\|_{1} \leq C\left\|g^{(N)}\right\|_{1 ; N},
$$

for a positive constant $C$ independent of $u^{(N)}$ and $g^{(N)}$ if $N$ is sufficiently large. Indeed, the equation (2.8)" and Lemma 2.1 imply

$$
\begin{aligned}
\left\|u^{(N)}\right\|_{1 ; N} & \leq\left\|H_{-} f_{+}^{-1} f_{-}^{-1}\right\|_{1}+\left\|g^{(N)} f_{+}^{-1} f_{-}^{-1}\right\|_{1}+\left\|H_{+} f_{+}^{-1} f_{-}^{-1}\right\|_{1} \\
& \leq\left\|f_{+}^{-1}\right\|_{1}\left\|H_{-} f_{-}^{-1}\right\|_{1}+\left\|f_{+}^{-1}\right\|_{1}\left\|f_{-}^{-1}\right\|_{1}\left\|g^{(N)}\right\|_{1 ; N}+\left\|f_{-}^{-1}\right\|_{1}\left\|H_{+} f_{+}^{-1}\right\|_{1} .
\end{aligned}
$$


Hence (2.9) follows from (2.10). Let $f_{ \pm}(z)^{-1}=\sum_{j \in \mathbf{N}} b_{ \pm j} z^{ \pm j} \in l_{1}^{ \pm}$, and put

$$
B_{ \pm}(N)=\sum_{j=N+1}^{\infty} b_{ \pm j} z^{ \pm j}
$$

Then $\left\|B_{ \pm}(N)\right\|_{1} \rightarrow 0$ as $N \rightarrow \infty$. The equation (2.8)" is rewritten in the form,

$$
u^{(N)}(z) f_{+}(z)=H_{-}(z) f_{-}(z)^{-1}+g^{(N)}(z) f_{-}(z)^{-1}+H_{+}(z) f_{-}(z)^{-1} .
$$

Looking at the coefficients of negative power of $z$ in this equation, we have

$$
H_{-}(z) f_{-}(z)^{-1}=-\left[g^{(N)} f_{-}^{-1}\right]_{-}-\left[H_{+} f_{-}^{-1}\right]_{-}=-\left[g^{(N)} f_{-}^{-1}\right]_{-}-\left[H_{+} B_{-}(N)\right]_{-},
$$

where $[u]_{-}:=(I-P) u \in l_{1}^{-}$for $u(z) \in l_{1}$. Hence we have

$$
\begin{aligned}
\left\|H_{-} f_{-}^{-1}\right\|_{1} & \leq\left\|g^{(N)} f_{-}^{-1}\right\|_{1}+\left\|H_{+} B_{-}(N)\right\|_{1} \\
& \leq\left\|f_{-}^{-1}\right\|_{1}\left\|g^{(N)}\right\|_{1 ; N}+\left\|B_{-}(N) f_{+}\right\|_{1}\left\|H_{+} f_{+}^{-1}\right\|_{1} .
\end{aligned}
$$

Since $\left\|B_{-}(N) f_{+}\right\|_{1} \leq\left\|B_{-}(N)\right\|_{1}\left\|f_{+}\right\|_{1} \rightarrow 0$ as $N \rightarrow \infty$, for any $0<\varepsilon<1$ there exists $N_{0}$ such that $\left\|B_{-}(N) f_{+}\right\|_{1}<\varepsilon$ for every $N \geq N_{0}$. Hence we have

$$
\left\|H_{-} f_{-}^{-1}\right\|_{1} \leq\left\|f_{-}^{-1}\right\|_{1}\left\|g^{(N)}\right\|_{1 ; N}+\varepsilon\left\|H_{+} f_{+}^{-1}\right\|_{1} \quad\left(N \geq N_{0}\right) .
$$

Next, we notice a relation,

$$
u^{(N)}(z) f_{-}(z)=H_{-}(z) f_{+}(z)^{-1}+g^{(N)}(z) f_{+}(z)^{-1}+H_{+}(z) f_{+}(z)^{-1},
$$

which follows from (2.8)". Then we have

$$
H_{+}(z) f_{+}(z)^{-1}=-\left[H_{-} f_{+}^{-1}\right]_{N}-\left[g^{(N)} f_{+}^{-1}\right]_{N}=-\left[H_{-} B_{+}(N)\right]_{N}-\left[g^{(N)} f_{+}^{-1}\right]_{N},
$$

where $[u]_{N}:=\sum_{j>N} u, z^{j}$. By taking large $N_{0}$ such that $\left\|B_{+}(N) f_{-}\right\|_{1}<\varepsilon$ holds for any $N \geq N_{0}$, we have

$$
\left\|H_{+} f_{+}^{-1}\right\|_{1} \leq\left\|f_{+}^{-1}\right\|_{1}\left\|g^{(N)}\right\|_{1 ; N}+\varepsilon\left\|H_{-} f_{-}^{-1}\right\|_{1} \quad\left(N \geq N_{0}\right) .
$$

Now we get (2.10) by taking $C=\left\{\left\|f_{+}^{-1}\right\|_{1}+\left\|f_{-}^{-1}\right\|_{1}\right\} /\left(1-\varepsilon^{2}\right)$. This proves the former half of the statement of the proposition.

Let us prove the latter half of the statement. We, first, prove the solvability of the equation, $T_{f} u=g \in l_{1}^{+}$.

For $g=\left(g_{0}, g_{1}, g_{2}, \ldots\right) \in l_{1}^{+}$we put $g^{(N)}=\left(g_{0}, g_{1}, \ldots, g_{N}\right) \in l_{1}[N]$. Let $N \geq N_{0}$ as in the norm inequality (2.9). There exists $u^{(N)}=\left(u_{0}^{(N)}, u_{1}^{(N)}, \ldots\right.$, $\left.u_{N}^{(N)}\right) \in l_{1}[N]$ satisfying $T_{f}(N) u^{(N)}=g^{(N)}$ and the norm inequality (2.9) holds. We identify $u^{(N)}$ with $u(N)=\left(u_{0}^{(N)}, u_{1}^{(N)}, \ldots, u_{N}^{(N)}, 0, \ldots\right) \in l_{1}^{+}$. It is the same for $g(N) \in l_{1}^{+}$. The norm inequality (2.9) implies 


$$
\|u(N)\|_{1} \leq K\|g(N)\|_{1} \leq K\|g\|_{1} .
$$

Then there exists $u=\left(u_{0}, u_{1}, u_{2}, \ldots\right) \in l_{1}^{+}$such that

$$
\lim _{N \rightarrow \infty} u_{j}^{(N)}=u_{j}(j \geq 0) \text {. }
$$

Strictly speaking, the limit $N \rightarrow \infty$ should be taken over a subsequence of $\mathbf{N}$, but we may assume as above without loss of generality.

Now it is proved that $u$ satisfies the equation, $T_{f} u=g$ in $l_{1}^{+}$. Indeed, we notice the equation,

$$
\sum_{j=0}^{N} f_{i-j} u_{j}^{(N)}=g_{i} \text { for } \quad 0 \leq i \leq N .
$$

By the definition of the Toeplitz symbol $f(z), f_{i-j}=0$ if $|i-j|>\max \{|m|,|n|\}$, and hence the summation is taken is over a fixed finite number of $j$ 's for any $i$. Therefore, we can take the limit $N \rightarrow \infty$ in the above equation, and we have

$$
\lim _{N \rightarrow \infty} \sum_{j=0}^{N} f_{i-j} u_{j}^{(N)}=\sum_{j=0}^{\infty} f_{i-j} u_{j}=g_{i},
$$

for any fixed $i$. This shows that $T_{f} u=g$ in $l_{1}^{+}$.

Next, we prove the uniqueness of solutions. Suppose $u \in l_{1}^{+}$satisfy $T_{f} u=0$. We take $N \geq N_{0}$, and rewrite the equation in the form,

$$
\sum_{j=0}^{N} f_{i-j} u_{j}=-\sum_{j=N+1}^{\infty} f_{i-j} u_{j} \stackrel{\text { put }}{=} g_{i}^{(N)}, \quad 0 \leq i \leq N .
$$

The norm inequality (2.9) shows

$$
\|u(N)\|_{1}=\left\|u^{(N)}\right\|_{1 ; N} \leq K\left\|g^{(N)}\right\|_{1 ; N},
$$

where $g^{(N)}=\left(g_{0}^{(N)}, g_{1}^{(N)}, \ldots, g_{N}^{(N)}\right)$. We remark again that the summations in both hand side are taken over a fixed finite number of $j$ 's, and $g_{i}^{(N)}=0$ for $0 \leq i<N$ $-\max \{|m|,|n|\}$. Hence we have

$$
\left\|g^{(N)}\right\|_{1 ; N} \leq C\|u-u(N)\|_{1},
$$

for a positive constant $C$ independent of $N$. Since $\|u-u(N)\|_{1} \rightarrow 0$ as $N \rightarrow \infty$, we have $\|u\|_{1}=\lim _{N \rightarrow \infty}\|u(N)\|_{1}=0$. This proves $u=0$ in $l_{1}^{+}$.

The proof is thus completed.

We remark that the condition $(\mathrm{H})_{w}$ implies the unique solvability of the $N$-th 
section Wiener-Hopf equation (2.8) only for sufficiently large $N$, and the condition does not control small $N$. For the invertibility of the $N$-th section Wiener-Hopf equations for all $N \in \mathbf{N}$, we have to impose other additional conditions, which will be given in the below.

Example 2.1 (cf. Example 1.2). Let $f(z)=\left(\frac{4}{3}-z^{-1}\right)\left(z+\frac{3}{2}\right)^{2}=\frac{4}{3} z^{2}+3 z$ $-\frac{9}{4} z^{-1}$. Then the condition $(\mathrm{H})_{w}$ is satisfied for $\frac{3}{4}<w<\frac{3}{2}$. The finite section Toeplitz matrices are given by

$$
T_{f}(0)=(0), T_{f}(1)=\left(\begin{array}{cc}
0 & -9 / 4 \\
3 & 0
\end{array}\right), T_{f}(2)=\left(\begin{array}{ccc}
0 & -9 / 4 & 0 \\
3 & 0 & -9 / 4 \\
4 / 3 & 3 & 0
\end{array}\right), \ldots
$$

It is easy to check that $\operatorname{det}\left(T_{f}(N)\right)>0$ for $N \geq 1$. Indeed, we have $\operatorname{det}\left(T_{f}(1)\right)=\operatorname{det}\left(T_{f}(2)\right)=27 / 4, \operatorname{det}\left(T_{f}(3)\right)=729 / 16$ and $\operatorname{det}\left(T_{f}(N)\right)=$ $(27 / 4)\left\{\operatorname{det}\left(T_{f}(N-2)\right)+\operatorname{det}\left(T_{f}(N-3)\right)\right\}>0$ for $N \geq 4$.

PROPOSITION 2.4. If one of the following conditions is satisfied, then $T_{f}(N)$ is invertible for every $N \in \mathbf{N}$ :

(i) $f(z)$ is a polynomial of $z$ or $z^{-1}$, and $(\mathrm{H})_{c}$ is satisfied for some $c>0$.

(ii) There exists $c>0$ such that $\left\{f(z) ; z \in K_{c}\right\}$ is a segment which does not contain the origin. In this case, the set of eigenvalues of $T_{f}(N)$ is included in this segment, and is densely distributed on this segment as $N \rightarrow \infty$ (Theorem of Szegö).

(iii) There exists $c>0$ such that $0 \notin \operatorname{ch}\left\{f(z) ; z \in K_{c}\right\}$.

Note that (ii) is a special case of (iii), but it has a special interest because of Szegö's precise theorem.

Proof. (i) It is obvious, since $T_{f}(N)$ are triangle matrices with non zero diagonal component $f_{0}$.

(ii) We take $\alpha, \beta \in \mathbf{C}(|\beta|=1)$ such that $h(z):=\beta\{\alpha-f(c z)\}$ is real valued on $|z|=1$. Then $h(z)$ is written in the Hermitian form,

$$
h(z)=h_{0}+\sum_{j=1}^{m}\left(h_{j} z^{j}+\bar{h}_{j} z^{-j}\right)
$$

where $h_{0} \in \mathbf{R}, h_{j} \in \mathbf{C}$ and $\bar{h}_{j}$ is its complex conjugate $(j \geq 1)$. Then we have

$$
D_{N}(c)\left\{\alpha I_{N+1}-T_{f}(N)\right\} D_{N}^{-1}(c)=\beta^{-1} T_{h}(N),
$$


where $I_{N+1}$ is the identity matrix of $\operatorname{order} N+1, D_{N}(c):=\operatorname{diag}\left\{1, c, \ldots, c^{N}\right\}$, the diagonal matrix with the $j$-th diagonal component $c^{j}(0 \leq j \leq N)$ and $D_{N}^{-1}(c)=D_{N}\left(c^{-1}\right)$.

Now our result follows from Szegö's theorem below.

Theorem [S, Satz XXII]. Let $h(z)$ be as above. Then we have

$$
\overline{\bigcup_{N} \sigma\left(T_{h}(N)\right)}=\{h(z) ;|z|=1\}
$$

where $\sigma\left(T_{h}(N)\right)$ denotes the set of eigenvalues of $T_{h}(N)$ and $\overline{\{\cdot\}}$ denotes the closure of a set of points.

(iii) It is a consequence of the following,

Lemma 2.5. If $\operatorname{Re} f(z)>0$ on $K_{c}$, then $\sigma\left(T_{f}(N)\right) \subset\{\lambda \in \mathbf{C} ; \operatorname{Re} \lambda>0\}$ for every $N \geq 0$.

Indeed, suppose this lemma and assume that $0 \notin \operatorname{ch}\left\{f(z) ; z \in K_{c}\right\}$. Then we can take $\alpha \in \mathbf{C}(|\alpha|=1)$ such that $\operatorname{Re}\{\alpha f(z)\}>0$ on $K_{c}$. Therefore, $\sigma\left(\alpha T_{f}(N)\right) \subset\{\lambda \in \mathbf{C} ; \operatorname{Re} \lambda>0\}$, and hence $T_{f}(N)$ is invertible for every $N \geq 0$.

Proof of Lemma 2.5. The $N$-th finite section matrix $T_{g}(N)$ of $g(z):=$ $f(c z)$ is given by $T_{g}(N)=D_{N}(c) T_{f}(N) D_{N}^{-1}(c)$. Let $u={ }^{t}\left(u_{0}, u_{1}, \ldots, u_{N}\right) \in \mathbf{C}^{N+1}$. Then we have

$$
\begin{aligned}
& \frac{1}{2 \pi} \int_{0}^{2 \pi}\left|u_{0}+u_{1} e^{i \theta}+\cdots+u_{N} e^{i N \theta}\right|^{2} g\left(e^{i \theta}\right) d \theta \\
& =\left(\bar{u}_{0}, \bar{u}_{1}, \ldots, \bar{u}_{N}\right) T_{g}(N)\left(\begin{array}{c}
u_{0} \\
u_{1} \\
\vdots \\
u_{N}
\end{array}\right)=: T_{g}(N)[u] .
\end{aligned}
$$

The assumption, $\operatorname{Re} g(z)>0$ on $K_{1}$, implies $\operatorname{Re} T_{g}(N)[u]>0$ on $\mathbf{C}^{N+1} \backslash\{0\}$. Hence $\sigma\left(T_{f}(N)\right)=\sigma\left(T_{g}(N)\right) \subset\{\lambda \in \mathbf{C} ; \operatorname{Re} \lambda>0\}$. This completes the proof.

\section{Spectral property of the principal part $L_{0}\left(D_{t}, D_{x}\right)$}

Recall that the principal part $L_{0}$ and the Toeplitz symbol $f(z)$ are given by

$$
L_{0}=\sum_{(j, \alpha) \in \stackrel{N}{N}_{s}} a_{0 j \alpha}(0) D_{t}^{j} D_{x}^{\alpha}, \quad f(z)=\sum_{(j, \alpha) \in \stackrel{N}{N}_{s}} a_{0 j \alpha}(0) z^{-j} .
$$


We put

$$
\begin{aligned}
& \Gamma_{w}^{+}(f):=\left\{\lambda \in \mathbf{C} ; f(z)-\lambda \text { satisfies }(\mathrm{H})_{w}\right\} \\
& \Gamma_{w}^{-}(f):=\left\{\lambda \in \mathbf{C} ; f(z)-\lambda \text { satisfies }(\mathrm{H})_{c w} \text { for } c=e^{s-1} \text { and } c=e^{s(s-1)}\right\} .
\end{aligned}
$$

Then the purpose of this section is to prove the following results.

Proposition 3.1 (The case $s>0$ ). For any fixed $R>0$, the operator $\lambda I-L_{0}$ is a Fredholm operator on $G_{w}^{s}(R)$ if and only if $\lambda \in \Gamma_{w}^{+}(f)$, and it is also necessary and sufficient that there exists $N$ such that $\lambda I-L_{0}$ is bijective on $G_{w}^{s}(R)[N]$. Here $G_{w}^{s}(R)[N]$ is the ideal of $G_{w}^{s}(R)$ defined in Theorem 1 . In this case, the operator norm of the inverse $\left(\lambda I-L_{0}\right)^{-1}$ is estimated uniformly on $R>0$, that is, there exists a constant $K>0$ such that $\left\|\left(\lambda I-L_{0}\right)^{-1}\right\| \leq K$ for every $R>0$. Moreover, for $\lambda \in$ $\Gamma_{w}^{+}(f)$ if $f(z)-\lambda$ satisfies one of the conditions in Proposition 2.4, then $\lambda \in \rho\left(L_{0}\right)$.

Proposition 3.2 (The case $s \leq 0$ ). Let $\lambda \in \Gamma_{w}^{-}(f)$. Then there exists $n_{0} \in \mathbf{N}$ such that $\lambda$ belongs to the resolvent set of $L_{0}$ on $G_{w}^{s}(R ; n)$ for every $n \geq n_{0}$ and every $R>0$. Moreover, we have a uniform estimate of $\left\|\left(\lambda I-L_{0}\right)^{-1}\right\|$ for $n \geq n_{0}$ and $R>0$.

First, we note that $(j, \alpha) \in \stackrel{\circ}{N}_{s}(s \in \mathbf{Q})$ if and only if $\alpha=-s j$. Let $s=$ $q / p(p, q \in \mathbf{Z}, p>0)$ be an irreducible fraction. Then the operator $L_{0}$ is rewritten in the form,

$$
L_{0}=\sum_{j=-m}^{n} f_{j} D_{t}^{j p} D_{x}^{-j q}\left(f_{-m} f_{n} \neq 0, m, n \in \mathbf{N}\right) \text {, }
$$

and the Toeplitz symbol $f(z)$ is rewritten by

$$
f(z)=\sum_{j=-m}^{n} f_{j} z^{-j p}
$$

We prove the propositions by setting $\lambda=0$, so we consider an equation,

$$
L_{0}\left(D_{t}, D_{x}\right) U(t, x)=F(t, x) .
$$

For $U(t, x) \in \mathbf{C}[[t, x]]$, we put $U(t, x)=\sum U_{l \beta} t^{l} x^{\beta} / l ! \beta !$. It is the same for $F(t, x) \in \mathbf{C}[[t, x]]$. Then the equation (3.4) gives the following equations for the coefficients $\left\{U_{l \beta}\right\}$ and $\left\{F_{l \beta}\right\}$.

$$
\sum_{j} f_{j} U_{l+p j, \beta-q j}=F_{l \beta}(-m \leq j \leq n ; l+p j, \beta-q j \in \mathbf{N}) .
$$


Proof of Proposition 3.1. In this case $q>0$, since $s>0$. We choose a lattice point $(l, \beta) \in \mathbf{N}^{2}$ such that $l-p<0$. We put $d(l, \beta)=\max \{j ; \beta-j q \geq 0\}$ $(\geq 0)$, and define a vector $\mathcal{U}^{(l, \beta)} \in \mathbf{C}^{d(l, \beta)+1}$ from $U(t, x) \in \mathbf{C}[[t, x]]$ by

$$
u^{(l, \beta)}={ }^{t}\left(u_{0}, u_{1}, \ldots, u_{d(l, \beta)}\right):={ }^{t}\left(U_{l, \beta}, U_{l+p, \beta-q}, \ldots, U_{l+d(l, \beta) p, \beta-d(l, \beta) q}\right) .
$$

Then for $U(t, x) \in G_{w}^{s}(R)$, we have

$$
\|U\|_{w, R}^{(s)}=\sum_{l, \beta}^{\prime}\left(\sum_{j=0}^{d(l, \beta)}\left|u_{j}\right| w^{j p}\right) \frac{w^{l} R^{s l+\beta}}{(s l+\beta) !},
$$

where $\sum_{l, \beta}^{\prime}$ is taken over such $l, \beta$ mentioned above. We remark that $s(l+j p)+$ $(\beta-j q)=s l+\beta$. This shows that $\mathcal{U}^{(l, \beta)} \in l_{1, w^{p}}[d(l, \beta)]$. We define $\mathscr{F}^{(l, \beta)}$ from $F(t, x)$ similarly.

Let $T_{l, \beta}$ be the $d(l, \beta)$-th finite section Toeplitz matrix with symbol $g(z):=$ $f\left(z^{1 / p}\right)=\sum_{j} f_{j} z^{-j}=\sum_{\text {put }} \sum_{j} z^{j}$, that is,

$$
T_{l, \beta}=\left(g_{j-k}\right)_{j, k=0,1, \ldots, d(l, \beta)} .
$$

Note that the condition $(\mathrm{H})_{w}$ is satisfied for $f(z)$ if and only if $(\mathrm{H})_{w^{p}}$ is satisfied for $g(z)$. The relation (3.5) implies the following equation for $\mathcal{U}^{(l, \beta)}$ and $\mathscr{F}^{(l, \beta)}$,

$$
T_{l, \beta} \mathcal{U}^{(l, \beta)}=\mathscr{F}^{(l, \beta)} \text {. }
$$

Hence, by (3.6), the operator $L_{0}$ on $G_{w}^{s}(R)$ is decomposed into an infinite direct product of the finite section Toeplitz matrices $T_{l, \beta}$ on $l_{1, w^{p}}[d(l, \beta)]$ (see Proposition 2.3).

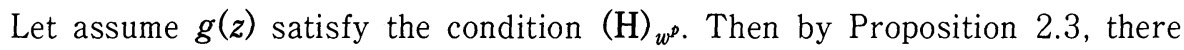
exists $N_{0}$ such that $T_{l, \beta}$ is invertible on $l_{1, w^{p}}[d(l, \beta)]$ for every $d(l, \beta) \geq N_{0}$, and the norm inequality (2.9) holds for a positive constant $K$ independent of $d(l, \beta) \geq$ $N_{0}$. This implies that $L_{0}$ is a Fredholm operator with an index 0 on $G_{w}^{s}(R)$, because $d(l, \beta) \rightarrow \infty$ as $\beta \rightarrow \infty$. The bijectivity of $L_{0}$ on $G_{w}^{s}(R)[N]$ for sufficiently large $N$ follows from the fact that $G_{w}^{s}(R)[N]$ is an infinite direct product of $l_{l, w^{\rho}}[k]$ of sufficiently large $k$ 's by the definition of $U^{(l, \beta)}$.

Next, suppose that $L_{0}$ is a Fredholm operator with an index 0 on $G_{w}^{s}(R)$. Then it follows that $\operatorname{det}\left(T_{l, \beta}\right) \neq 0$ if $d(l, \beta)$ is sufficiently large. Indeed, if not so, $L_{0}$ should have infinite dimensional kernel and cokernel by the decomposition of finite section Toeplitz matrices, which is a contradiction. Therefore, $L_{0}$ maps $G_{w}^{s}(R)[N]$ into itself, and is injective for sufficiently large $N$. We fix such a large $N$. The following exact sequence of Banach spaces

$$
0 \rightarrow G_{w}^{s}(R)[N] \rightarrow G_{w}^{s}(R) \rightarrow G_{w}^{s}(R) / G_{w}^{s}(R)[N] \rightarrow 0,
$$


and the additivity of indices imply that $L_{0}$ is a Fredholm operator with an index 0 on $G_{w}^{s}(R)[N]$, and therefore bijective. This means that $L_{0}$ has a bounded inverse on $G_{w}^{s}(R)[N]$. This implies that $T_{l, \beta}$ is invertible on $l_{1, w^{p}}[d(l, \beta)]$ with uniform norm inequality (2.9) for sufficiently large $d(l, \beta)$. This shows that $g(z)$ satisfies the condition $(\mathrm{H})_{w^{p}}$.

We note that if $f(z)$ satisfies $(\mathrm{H})_{w}$ and also satisfies one of the conditions in Proposition 2.4, then $T_{l, \beta}$ is invertible for every $l, \beta$ and the norm inequality (2.9) holds for every $l, \beta$. This implies the latter half of the statement.

The proof is thus completed.

Remark 3.1. The decomposition of the operator $L_{0}$ into an infinite direct product of the finite section Toeplitz matrices $\left\{T_{l, \beta}\right\}$ implies the following fact; if $\{f(z) ;|z|=c\}$ is a segment for some $c>0$, then by Szegö's theorem in Section 2 we have

$$
\sigma_{p}\left(L_{0}\right)=\bigcup_{l, \beta} \sigma\left(T_{l, \beta}\right),
$$

where $\sigma_{p}\left(L_{0}\right)$ (resp. $\sigma\left(T_{l, \beta}\right)$ ) denotes the set of eigenvalues of $L_{0}$ on $G_{w}^{s}(R)$ (resp. of $T_{l, \beta}$ ). This proves the fact stated in Remark 1.2, (ii).

Proof of Proposition 3.2. The assumption $s \leq 0$ implies that the equations (3.5) give a sequence of infinite systems of linear equations, since $q \leq 0$. For $(l, \beta) \in \mathbf{N}^{2}$ such that $l-p<0$ or $\beta+q<0$, we define $\mathcal{U}^{(l, \beta)} \in \mathbf{C}^{\infty}$ by

$$
U^{(l, \beta)}={ }^{t}\left(U_{l \beta}, U_{l+p, \beta-q}, U_{l+2 p, \beta-2 q}, \ldots\right) \stackrel{\text { put }}{=}\left(U_{0}^{(l, \beta)}, U_{1}^{(l, B)}, U_{2}^{(l, \beta)}, \ldots\right) .
$$

We define $\mathscr{F}^{(l, \beta)}$ similarly. Then the equations (3.5) give the following infinite equations for $\mathcal{U}^{(l, \beta)}$ and $\mathscr{F}^{(l, \beta)}$,

$$
T_{g} U^{(l, \beta)}=\mathscr{F}^{(l, \beta)},
$$

where $T_{g}$ is the Toeplitz matrix with the symbol $g(z):=f\left(z^{1 / p}\right)$. Since $f(z)$ satisfies the condition $(\mathrm{H})_{c w}$ for $c=e^{s-1}$ and $c=e^{s(s-1)}, g(z)$ satisfies the condition $(\mathrm{H})_{c}$ for $c=w^{p} e^{p(s-1)}$ and $c=w^{p} e^{p s(s-1)}$. Hence, by Proposition 2.2, $T_{g}$ is a bijection on $l_{\infty, r}^{+}$for every $r$ in a neighbourhood of a closed interval $\left[w^{p} e^{p(s-1)}\right.$, $\left.w^{p} e^{p s(s-1)}\right]$. Precisely, let

$$
T_{g}^{-1}:=\left(c_{j k}\right)_{j, k=0,1,2, \ldots} .
$$

Then there exists a constant $\varepsilon>0$ such that 


$$
\left\|T_{g}^{-1}\right\|=\sup _{j} \sum_{k=0}^{\infty}\left|c_{j k}\right| w^{p(j-k)} r^{j-k}<\infty,
$$

for every $r$ such that $e^{p(s-1)}-\varepsilon \leq r \leq e^{p s(s-1)}+\varepsilon$.

We note that $g(z)$ satisfies $(\mathrm{H})_{w^{p}}$ because $s \leq 0$, and hence $T_{g}$ is a bijection on $l_{\infty, r}^{+}$for $r<w^{p}$ sufficiently near $w^{p}$. By the definition of the norm in $G_{w}^{s}(R ; n)$, $U(t, x) \in G_{w}^{s}(R ; n)$ if and only if there exists a non negative constant $C$ such that

$$
w^{p \jmath}\left|U_{j}^{(l, \beta)}\right| \leq C \frac{w_{l, \beta ; n}(j)}{w^{l} R^{s l+\beta}}, j \geq 0,
$$

for every $(l, \beta)$ satisfying the above mentioned condition. Here

$$
w_{l, \beta ; n}(j):=\frac{\{n l+(n+1) \beta+j p(n-(n+1) s)\} !}{\{(n-s) l+n \beta+j p(n-(n+1) s)\} !} \quad(j \geq 0) .
$$

Moreover it holds that $\|U\|_{w, R, n}^{(s)}=\inf \{C ; C$ satisfies $(3.11)\}$. Note that $w_{l, \beta ; n}(j)$ has polynomial increase or decrease in $j$ according to the position of $(l, \beta)$. In any case, $U^{(l, \beta)} \in l_{\infty, r}^{+}\left(r<w^{p}\right)$ whenever $U(t, x) \in G_{w}^{s}(R ; n)$. Therefore, for any $F(t, x) \in G_{w}^{s}(R ; n)$, the equation (3.9) has a unique solution $\mathcal{U}^{(l, \beta)} \in l_{\infty, r}^{+}$for $r<w^{p}$ sufficiently near $w^{p}$.

In order to prove that $L_{0}$ is bijective on $G_{w}^{s}(R ; n)$ with uniform estimate of $\left\|L_{0}^{-1}\right\|(R>0)$ for sufficiently large $n$, we put

$$
D_{l \beta}=\operatorname{diag}\left\{w_{l, \beta ; n}(j)\right\}_{j=0}^{\infty},
$$

a diagonal matrix with the $j$-th diagonal component $w_{l, \beta ; n}(j)$. Then we have that

$$
w^{l} R^{s l+\beta} D_{l \beta}^{-1} U^{(l, \beta)} \in l_{\infty, w^{p}}^{+},
$$

if (3.11) is satisfied. Hence, in view of (3.9), it is sufficient to show that the operator norm of $D_{l \beta}^{-1} T_{g}^{-1} D_{l \beta}$ on $l_{\infty, w^{p}}^{+}$is uniformly bounded on $(l, \beta)$. This is equivalent to prove

$$
\sup _{l, \beta} \sup _{j} \sum_{k=0}^{\infty}\left|c_{j k}\right| w^{p(j-k)} w_{l, \beta ; n}(j)^{-1} w_{l, \beta ; n}(k)<\infty .
$$

From (3.10) with $r=e^{p s(s-1)}+\varepsilon$ for $k \leq j$ and $r=e^{p(s-1)}-\varepsilon$ for $k>j$, it is sufficient to prove that for any $\varepsilon>0$ there exists $n_{0} \in \mathbf{N}$ such that

$$
w_{l, \beta ; n}(j)^{-1} w_{l, \beta ; n}(k)<\left\{\begin{array}{l}
\left(e^{p s(s-1)}+\varepsilon\right)^{j-k}, 0 \leq k \leq j, \\
\left(e^{p(1-s)}+\varepsilon\right)^{k-j}, 0 \leq j<k,
\end{array}\right.
$$


holds for $n \geq n_{0}$. This is a consequence of the following,

Lemma 3.3. Let $w_{l, \beta ; n}(j)$ be as above. Then we have

$$
w_{l, \beta ; n}(j)^{-1} w_{l, \beta ; n}(k) \leq\left\{\begin{array}{l}
e^{-s p(1-s(1+1 / n))(j-k)}, 0 \leq k \leq j, \\
e^{p(1-s(1+1 / n))(k-j)}, 0 \leq j<k .
\end{array}\right.
$$

Proof. In the case $0 \leq k \leq j$, we have

$$
\begin{aligned}
w_{l, \beta ; n}(j)^{-1} & w_{l, \beta ; n}(k) \\
& =\prod_{r=1}^{(n-(n+1) s) p(j-k)} \frac{(n-s) l+n \beta+(n-(n+1) s) p k+r}{n l+(n+1) \beta+(n-(n+1) s) p k+r} \\
& \leq\left(\frac{n-s}{n}\right)^{\{n-(n+1) s\} p(j-k)} \leq e^{-s(1-(1+1 / n) s\} p(j-k)} .
\end{aligned}
$$

In the case $0 \leq j<k$, we have

$$
\begin{aligned}
w_{l, \beta ; n}(j)^{-1} & w_{l, \beta ; n}(k) \\
& =\prod_{r=1}^{(n-(n+1) s) p(k-j)} \frac{n l+(n+1) \beta+(n-(n+1) s) p j+r}{(n-s) l+n \beta+(n-(n+1) s) p j+r} \\
& \leq\left(\frac{n+1}{n}\right)^{\{n-(n+1) s\} p(k-j)} \leq e^{\{1-(1+1 / n) s\} p(k-j)} .
\end{aligned}
$$

This completes the proof of Proposition 3.2.

\section{Proofs of Theorems 1 and 2}

Theorems are proved by stability theorems of Fredholm operators.

We denote by $\mathscr{O}(|x| \leq X)$ the set of holomorphic functions on $\{x \in \mathbf{C} ;|x|$ $<X\}$ and continuous on its closure. For $a(x) \in \mathscr{O}(|x| \leq X)$, we put

$$
\|a\|_{X}:=\max _{|x| \leq X}|a(x)| \text {. }
$$

The following lemmas are proved essentially in [M2] and [MH] with a slight change of notations.

Lemma 4.1. (i) Let $s>0$. If $a(x) \in \mathscr{O}(|x| \leq \rho R)(\rho>1)$, then for any $U(t, x) \in G_{w}^{s}(R)$, we have $a(x) U(t, x) \in G_{w}^{s}(R)$ and 


$$
\|a U\|_{w, R}^{(s)} \leq \frac{\rho}{\rho-1}\|a\|_{\rho R}\|U\|_{w, R}^{(s)}
$$

(ii) Let $s \leq 0$. If $a(x) \in \mathscr{O}(|x| \leq \rho R)\left(\rho>e^{-s}\right)$, then for any $U(t, x) \in$ $G_{w}^{s}(R ; n)$, we have $a(x) U(t, x) \in G_{w}^{s}(R ; n)$ and

$$
\|a U\|_{w, R, n}^{(s)} \leq \frac{\rho}{\rho-e^{-s}}\|a\|_{\rho R}\|U\|_{w, R, n}^{(s)}
$$

Proof. We put $a(x)=\sum a_{\gamma} x^{\gamma} / \gamma ! \in \mathscr{O}(|x| \leq \rho R)$. They by Cauchy's integral formula, we have $\left|a_{\gamma}\right| \leq\|a\|_{p R} \gamma ! /(\rho R)^{\gamma}(\gamma \in \mathbf{N})$. We put $a(x) U(t, x)$ $=\sum V_{l \beta} t^{l} x^{\beta} / l ! \beta !$. Then we have

$$
V_{l \beta}=\sum_{\gamma=0}^{\beta} a_{\gamma} U_{l, \beta-\gamma} \frac{\beta !}{(\beta-\gamma) ! \gamma !} .
$$

(i) The inequality (4.1) is obtained as follows.

$$
\begin{aligned}
\sum_{l, \beta \in \mathbf{N}}\left|V_{l \beta}\right| & \frac{w^{l} R^{s l+\beta}}{(s l+\beta) !} \leq\|a\|_{\rho R} \sum_{l, \beta} \sum_{\gamma=0}^{\beta}\left|U_{l, \beta-\gamma}\right| \frac{1}{(\rho R)^{\gamma}} \frac{\beta !}{(\beta-\gamma) !} \frac{w^{l} R^{s l+\beta}}{(s l+\beta) !} \\
& \leq\|a\|_{\rho R} \sum_{l, \beta} \sum_{\gamma=0}^{\infty}\left|U_{l, \beta-\gamma}\right| \frac{1}{\rho^{\gamma}} \frac{w^{l} R^{s l+\beta-\gamma}}{(s l+\beta-\gamma) !} \\
& =\|a\|_{\rho R} \sum_{\gamma=0}^{\infty} \frac{1}{\rho^{\gamma}}\left(\sum_{l=0}^{\infty} \sum_{\beta=\gamma}^{\infty}\left|U_{l, \beta-\gamma}\right| \frac{w^{l} R^{s l+\beta-\gamma}}{(s l+\beta-\gamma) !}\right) \\
& =\|a\|_{\rho R}\|U\|_{w, R}^{(s)} \sum_{\gamma=0}^{\infty} \rho^{-\gamma}=\frac{\rho}{\rho-1}\|a\|_{\rho R}\|U\|_{w, R}^{(s)} .
\end{aligned}
$$

(ii) By the definition of the norm, we have

$$
\begin{aligned}
& \left|V_{l \beta}\right| w^{l} R^{s l+\beta} \frac{((n-s) l+n \beta) !}{(n l+(n+1) \beta) !} \leq\|a\|_{\rho R}\|U\|_{w, R, n}^{(s)} \\
& \quad \times \sum_{r=0}^{\beta} \rho^{-\gamma} \frac{\beta !}{(\beta-\gamma) !} \frac{((n-s) l+n \beta) !}{((n-s) l+n(\beta-\gamma)) !} \frac{(n l+(n+1)(\beta-\gamma)) !}{(n l+(n+1) \beta) !}
\end{aligned}
$$

Now (4.2) follows from the following inequality,

$$
\begin{aligned}
& \frac{\beta !}{(\beta-\gamma) !} \frac{((n-s) l+n \beta) !}{((n-s) l+n(\beta-\gamma)) !} \frac{(n l+(n+1)(\beta-\gamma)) !}{(n l+(n+1) \beta) !} \\
& =\prod_{j=1}^{n \gamma} \frac{(n-s) l+n(\beta-\gamma)+j}{n l+(n+1)(\beta-\gamma)+j} \prod_{j=1}^{r} \frac{\beta-\gamma+j}{n l+(n+1) \beta-\gamma+j}
\end{aligned}
$$




$$
\leq\left(\frac{n-s}{n}\right)^{n r} \leq e^{-s r}
$$

This completes the proof.

Lemma 4.2. If $s \geq 1$, then $G_{w}^{s}(R)$ is a Banach algebra. Precisely, for $a(t, x)$, $U(t, x) \in G_{w}^{s}(R)$, we have $a(t, x) U(t, x) \in G_{w}^{s}(R)$ and

$$
\|a U\|_{w, R}^{(s)} \leq\|a\|_{w, R}^{(s)}\|U\|_{w, R}^{(s)}
$$

Proof. We put $a(t, x)=\sum a_{j \alpha} t^{j} x^{\alpha} / j ! a !, U(t, x)=\sum U_{l \beta} t^{l} x^{\beta} / l ! \beta !$ and $a(t, x) U(t, x)=\sum V_{l \beta} t^{l} x^{\beta} / l ! \beta !$. Then we have

$$
V_{l \beta}=\sum_{j=0}^{l} \sum_{\alpha=0}^{\beta} a_{j \alpha} U_{l-j, \beta-\alpha} \frac{l !}{j !(l-j) !} \frac{\beta !}{\alpha !(\beta-\alpha) !} .
$$

Suppose the following inequality which is trivial in the case $s=1$,

$$
\frac{(s j+\alpha) !(s(l-j)+\beta-\alpha) !}{(s l+\beta) !} \frac{l !}{j !(l-j) !} \frac{\beta !}{\alpha !(\beta-\alpha) !} \leq 1
$$

Then we have

$$
\begin{aligned}
\sum_{l, \beta \in \mathbf{N}} \mid & V_{l \beta} \mid \frac{w^{l} R^{s l+\beta}}{(s l+\beta) !} \\
& \leq \sum_{l, \beta} \sum_{j=0}^{l} \sum_{\alpha=0}^{\beta}\left|a_{j \alpha}\right| \frac{w j R^{s j+\alpha}}{(s j+\alpha) !}\left|U_{l-j, \beta-\alpha}\right| \frac{w^{l-j} R^{s(l-j)+(\beta-\alpha)}}{(s(l-j)+(\beta-\alpha)) !} \\
& =\sum_{j, \alpha}\left(\sum_{l \geq j, \beta \geq \alpha}\left|U_{l-j, \beta-\alpha}\right| \frac{w^{l-j} R^{s(l-j)+(\beta-a)}}{(s(l-j)+(\beta-\alpha)) !}\right)\left|a_{j \alpha}\right| \frac{w^{j} R^{s j+\alpha}}{(s j+\alpha) !} \\
& =\|a\|_{w, R}^{(s)}\|U\|_{w, R}^{(s)} .
\end{aligned}
$$

Let us prove the inequality (4.4) in case $s>1$. Considering the inequalities,

$$
\begin{aligned}
& \frac{l !}{j !(l-j) !} \frac{\beta !}{\alpha !(\beta-\alpha) !} \leq \frac{(l+\beta) !}{(j+\alpha) !(l+\beta-j-\alpha) !}, \\
& \frac{(s(l-j)+\beta-\alpha) !(l+\beta) !}{(l+\beta-j-\alpha) ! \alpha !} \leq(s l+\beta-(s-1) j) !,
\end{aligned}
$$

we have

$$
(s j+\alpha) !(s(l-j)+\beta-\alpha) ! \frac{l !}{j !(l-j) !} \frac{\beta !}{\alpha !(\beta-\alpha) !}
$$




$$
\leq(s l+\beta-(s-1) j) ! \frac{(s l+\alpha) !}{(j+\alpha) !} .
$$

Recall the relation between gamma function and beta function, $\Gamma(x+y)$. $B(x, y)=\Gamma(x) \Gamma(y)(x, y>0)$. Then we have

$\frac{(s j+\alpha) !}{(j+\alpha) !}=\frac{\Gamma((s-1) j)}{B((s-1) j, j+\alpha+1)}$,

$\Gamma((s-1) j)(s l+\beta-(s-1) j) !=(s l+\beta) ! B((s-1) j, s l+\beta-(s-1) j+1)$.

These imply

$$
\begin{aligned}
& (s j+\alpha) !(s(l-j)+\beta-\alpha) ! \frac{l !}{j !(l-j) !} \frac{\beta !}{\alpha !(\beta-a) !} \\
& \leq(s l+\beta) ! \frac{B((s-1) j, s l+\beta-(s-1) j+1)}{B((s-1) j, j+\alpha+1)} \leq(s l+\beta) !,
\end{aligned}
$$

because $s l+\beta-(s-1) j+1 \geq j+\alpha+1$, since $s>1$. This proves (4.4).

Lemma 4.3. Let $(\sigma, j, \alpha) \in \mathbf{N} \times \mathbf{Z} \times \mathbf{Z}$ satisfy

$$
s j+(1-s) \sigma+\alpha=-\delta \leq 0 .
$$

Then $t^{\sigma} D_{t}^{j} D_{x}^{\alpha}$ is a bounded operator on $G_{w}^{s}(R)$ or on $G_{w}^{s}(R ; n)$ according to $s>0$ and $s \leq 0$, and the operator norm is estimated by

$$
\left\|t^{\sigma} D_{t}^{j} D_{x}^{\alpha}\right\| \leq C w^{\sigma-j} R^{\sigma+\delta},
$$

for some positive constant $C=C(\sigma, j, \alpha, s, n)$ independent of $R$. Moreover, if $\delta>0$, then $t^{\sigma} D_{t}^{j} D_{x}^{\alpha}$ is a compact operator on each Banach space.

Proof. We only prove the case $s>0$, since it is similar in the case $s \leq 0$. We put $t^{\sigma} D_{t}^{j} D_{x}^{\alpha} U(t, x)=\sum V_{l \beta} t^{l} x^{\beta} / l ! \beta !$. Then we have

$$
V_{l \beta}=\frac{l !}{(l-\sigma) !} U_{l+j-\sigma, \beta+\alpha} .
$$

This implies immediately that

$$
\left\|t^{\sigma} D_{t}^{j} D_{x}^{\alpha} U\right\|_{w, R}^{(s)} \leq C(\sigma, j, \alpha, s) w^{\sigma-j} R^{\sigma+\delta}\|U\|_{w, R}^{(s)},
$$

where $\quad C(\sigma, j, \alpha, s)=\sup \{(s l+\beta-\sigma-\delta) ! l ! /(s l+\beta) !(l-\sigma) ! ; l, \beta \in \mathbf{N}\}$. This implies the estimate (4.6). Let $\delta>0$. Then for any $\varepsilon>0$, there exists $N$ such that 


$$
\sup \left\{\frac{(s l+\beta-\sigma-\delta) ! l !}{(s l+\beta) !(l-\sigma) !} ; s l+\beta \geq N\right\}<\varepsilon
$$

Hence we have,

$$
\sum_{s l+\beta \geq N}\left|V_{l \beta}\right| \frac{w^{l} R^{s l+\beta}}{(s l+\beta) !}<\varepsilon w^{\sigma-j} R^{\sigma+\delta}\|U\|_{w, R}^{(s)} .
$$

This shows that $t^{\sigma} D_{t}^{j} D_{x}^{\alpha}$ is approximated by operators of finite rank, and therefore a compact operator. This completes the proof.

Recall the decomposition (1.3) of the operator $L$,

$$
L=L_{0}+L_{1}+L_{2}
$$

where $L_{0}$ is the principal part and

$$
\begin{aligned}
& L_{1}=\sum_{s j+(1-s) \sigma+\alpha=0} a_{\sigma j \alpha}(x) t^{\sigma} D_{t}^{j} D_{x}^{\alpha}\left(a_{0 j \alpha}(0)=0 \text { or } \sigma>0\right), \\
& L_{2}=\sum_{s j+(1-s) \sigma+\alpha<0}^{\text {finite }} a_{\sigma j \alpha}(t, x) t^{\sigma} D_{t}^{j} D_{x}^{\alpha} .
\end{aligned}
$$

From the above lemmas, $L_{2}$ is a compact operator on each Banach space $G_{w}^{s}(R)$ or $G_{w}^{s}(R ; n)$ according $s>0$ or $s \leq 0$. For the operator norms of $L_{j}(j=1,2)$ on each space, we have

$$
\left\|L_{j}\right\| \rightarrow 0 \text { as } R \rightarrow 0
$$

Proof of Theorem 1. (i) Let the Toeplitz symbol $f(z)$ satisfy the condition $(\mathrm{H})_{w}$. Then by Proposition 3.1 , there exists $N \in \mathbf{N}$ such that $L_{0}$ is bijective on $G_{w}^{s}(R)[N]$ and the norm of inverse operator $L_{0}^{-1}$ is estimated uniformly on $R>0$, that is, $\left\|L_{0}^{-1}\right\| \leq K$ for some $K>0$ and every $R>0$. Therefore, by (4.8) there exists $R_{0}>0$ such that $L$ is bijective on $G_{w}^{s}(R)[N]$ for every $0<R<R_{0}$. Now, by the same way as the proof of Proposition 3.1, we can prove that $L$ is a Fredholm operator on $G_{w}^{s}(R)$ for every $0<R<R_{0}$. This proof shows that if $L_{0}$ is bijective on $G_{w}^{s}(R)$, then there exists a positive constant $R_{0}>0$ such that $L$ is bijective on $G_{w}^{s}(R)$ for every $0<R \leq R_{0}$.

Next, we study the operator $L$ on $\mathcal{M}^{s}[N]$. By the definition of $\mathcal{M}^{s}[N], L$ maps $\mathcal{M}^{s}[N]$ into itself. Precisely, for $U(t, x)=\sum U_{l \beta} t^{l} x^{\beta} / l ! \beta ! \in \mathbf{C}[[t, x]]$, we put $r(U):=\min \left\{s l+\beta ; U_{l \beta} \neq 0\right\}$. Then we have

$$
r\left(L_{j} U\right)>r(U), \quad j=1,2 .
$$


Therefore, by the proof of Proposition 3.1, we see that if $\operatorname{det}\left(T_{l, \beta}\right) \neq 0$ for $s l+\beta$ $\geq N$, then there exists a recurrence formula to obtain a unique solution in $\mathcal{M}^{s}[N]$ of an equation, $L U(t, x)=F(t, x) \in \mathcal{M}^{s}[N]$. This implies that $L$ is bijective on $\mathcal{M}^{s}[N]$ and also on $G_{w}^{s}(R)[N]$ for sufficiently large $N$ and small $R>0$ under the condition $(\mathrm{H})_{w}$. The bijectivity of $L$ on $\left.\mathbf{C}[t, x]\right] / G_{w}^{s}(R)$ for sufficiently small $R>0$ is now obvious, because $\mathbf{C}[[t, x]] / G_{w}^{s}(R) \cong \mathcal{M}^{s}[N] / G_{w}^{s}(R)[N]$ for every $N$.

(ii) Let $L_{1}=0$ in the decomposition of the operator $L$. Suppose that $L$ be a Fredholm operator on $G_{w}^{s}(R)$ for some $R>0$. Then $L_{0}=L-L_{2}$ is also a Fredholm operator, since $L_{2}$ is a compact operator. Hence the condition $(\mathrm{H})_{w}$ is satisfied.

Next, suppose $\lambda \in \rho\left(L ; G_{w}^{s}(R)\right)$ for some $R>0$. Then $\lambda I-L_{0}$ is a Fredholm operator on $G_{w}^{s}(R)$ as above, and hence $\lambda \in \Gamma_{w}^{+}(f)$. Therefore, there exists $N$ such that $\lambda I-L_{0}$ and also $\lambda I-L$ are bijective on $G_{w}^{s}(R)[N]$. Here we note that the operator norm of $L_{2}$ on $G_{w}^{s}(R)[N]$ tends to 0 as $N \rightarrow \infty$ from the proof of Lemma 4.3. The exact sequence of Banach spaces,

$$
0 \rightarrow G_{w}^{s}(R)[N] \rightarrow G_{w}^{s}(R) \rightarrow G_{w}^{s}(R) / G_{w}^{s}(R)[N] \rightarrow 0
$$

implies that an induced operator from $\lambda I-L$ on $G_{w}^{s}(R) / G_{w}^{s}(R)[N]$ ( $\cong$ finite dimensional vector space) is bijective. The matrix representation of this operator is a blockwise triangle matrix with diagonal blocks of the Toeplitz matrices $\lambda I-$ $T_{l, \beta}$. Hence, we have $\operatorname{det}\left(\lambda I-T_{l, \beta}\right) \neq 0$ for every $l, \beta$. This shows $\lambda \in \rho\left(L_{0}\right)$, that is,

$$
\rho\left(L_{0}\right) \supset \bigcup_{R>0} \rho\left(L ; G_{w}^{s}(R)\right) .
$$

The converse relation is obvious. These prove (1.8).

Thus the proof of Theorem 1 is completed.

Proof of Theorem 2. It is almost obvious, so we omit it.

\section{Proof of Theorem 0}

Theorem 0 is a consequence of Theorem 1 and the following proposition.

Proposition 5.1. For $s>0$, it holds that

$$
\bigcap_{0<r<R} G_{w}^{s}(r)=\mathscr{G}_{w}^{s}(R) .
$$


Proof. For $U(t, x)=\sum_{l, \beta} U_{l \beta} t^{l} x^{\beta} / l ! \beta ! \in G_{w}^{s}(r)$, let us prove

$$
\tilde{U}(t, x):=\sum U_{l \beta} t^{l} x^{\beta} /(s l) ! \beta ! \in \mathscr{O}\left(\left(\frac{|t|}{w}\right)^{1 / s}+|x|<r\right),
$$

which implies $\cap_{0<r<R} G_{w}^{s}(r) \subset \mathscr{G}_{w}^{s}(R)$. By the definition of the norm in $G_{w}^{s}(R)$, there exists a positive constant $M$ such that $\left|U_{l \beta}\right| \leq M(s l+\beta) ! / w^{l} r^{s l+\beta}(l, \beta \in$ N). This implies

$$
\begin{aligned}
U_{l}(x) & :=\sum_{\beta} U_{l \beta} \frac{x^{\beta}}{\beta !} \ll M w^{-l} r^{-s l} \sum_{\beta} \frac{(s l+\beta) !}{\beta !} \frac{x^{\beta}}{r^{\beta}} \\
& =M w^{-l} r^{-s l} \frac{(s l) !}{(1-x / r)^{s l+1}}=M \frac{w^{-l}}{(1-x / r)} \frac{(s l) !}{(r-x)^{s l}},
\end{aligned}
$$

where $\sum_{\beta \in \mathbf{N}} a_{\beta} x^{\beta} \ll \sum A_{\beta} x^{\beta}$ means that $\left|a_{\beta}\right| \leq A_{\beta}$ for all $\beta \in \mathbf{N}$. Then we have

$$
\tilde{U}(t, x) \ll M \frac{1}{(1-x / r)} \sum_{l=0}^{\infty} \frac{1}{(r-x)^{s l}}\left(\frac{t}{w}\right)^{l},
$$

and the right hand side belongs to $\mathscr{O}\left((|t| / w)^{1 / s}+|x|<r\right)$.

Conversely, let $\tilde{U}(t, x)=\sum \tilde{U}_{l \beta} t^{l} x^{\beta} / l ! \beta ! \in \mathscr{O}\left((|t| / w)^{1 / s}+|x| \leq r\right)$. Then we shall prove

$$
U(t, x):=\sum_{l, \beta}\left\{\tilde{U}_{l \beta} \frac{(s l) !}{l !}\right\} \frac{t^{l} x^{\beta}}{l ! \beta !} \in G_{w}^{s}(\kappa r)
$$

for any $0<\kappa<1$. We denote by $\|\tilde{U}\|_{\infty}$ the maximum of $|\tilde{U}(t, x)|$ on the domain. Let $0<\tau<w r^{s}$, and put $\xi=r-(\tau / w)^{1 / s}$. By Cauchy's integral formula, we have

$$
\tilde{U}_{l \beta}=\frac{l ! \beta !}{(2 \pi i)^{2}} \oint_{|t|=\tau} \oint_{|x|=\xi} \frac{\tilde{U}(t, x)}{t^{l+1} x^{\beta+1}} d t d x .
$$

This implies

$$
\left|\tilde{U}_{l \beta}\right| \leq\|\tilde{U}\|_{\infty} \frac{l ! \beta !}{\tau^{l}\left(r-(\tau / w)^{1 / s}\right)^{\beta}} .
$$

The function $f(\tau):=\tau^{-l}\left\{r-(\tau / w)^{1 / s}\right\}^{-\beta}\left(0<\tau<w r^{s}\right)$ takes the minimum value

$$
w^{-l} \frac{(s l+\beta)^{s l+\beta}}{r^{s l+\beta}(s l)^{s l} \beta^{\beta}}
$$


at $\tau=w\{s l r /(s l+\beta)\}^{s}$. Put $U_{l \beta}=\tilde{U}_{l \beta}(s l) ! / l !$. Then we have

$$
\left|U_{l \beta}\right| \leq\|\tilde{U}\|_{\infty} \frac{(s l+\beta)^{s l+\beta}(s l) ! \beta !}{w^{l} r^{s l+\beta}(s l)^{s l} \beta^{\beta}}
$$

From Stirling's formula, we see that it holds that

$$
\frac{(s l+\beta)^{s l+\beta}}{(s l+\beta) !} \frac{(s l) !}{(s l)^{s l}} \frac{\beta !}{\beta^{\beta}} \sim \sqrt{2 \pi} \sqrt{\frac{s l \beta}{s l+\beta}} \quad \text { as } \quad s l+\beta \rightarrow \infty .
$$

Hence we have

$$
\|U\|_{w, \alpha r}^{(s)}=\sum_{l, \beta}\left|U_{l \beta}\right| \frac{w^{l}(\kappa r)^{s l+\beta}}{(s l+\beta) !}<\infty
$$

for any $0<\kappa<1$. This completes the proof.

\section{REFERENCES}

[B] G. Baxter, A norm inequality for a "finite section" Wiener-Hopf equation, Illinois J. Math., 7 (1963), 97-103.

[CSW] A. Calderón, F. Spitzer and H. Widom, Inversion of Toeplitz matrices, Illinois J. Math., 3 (1959), 490-498.

[G] L. Gårding, Une variante de la méthode de majoration de Cauchy, Acta Math., 114 (1965), 143-158.

[GS] U. Grenander and G. Szeg̈o, Toeplitz forms and their applications, Chelsea Publ., New York, 1984.

[H] L. Hörmander, Linear partial differential operators, Springer Verlag, Berlin, New York, 1963.

[L] L. Leray, Caractère non fredholmien du problème de Goursat, J. Math. Pures Appl., 53 (1974), 133-136.

[LP] J. Leray et C. Pisot, Une fonction de la théorie des nombres, J. Math. Pures Appl., 53 (1974), 137-145.

[Ma] B. Malgrange, Sur les points singuliers des équations différentielles linéaires, Enseign. Math., 20 (1970), 146-176.

[M1] M. Miyake, Global and local Goursat problems in a class of holomorphic or partially holomorphic functions, J. Differential Equations, 39 (1981), 445-463.

[M2] - Newton polygons and formal Gevery indices in the Cauchy-GoursatFuchs type equations, J. Math. Soc. Japan, 43 (1991), 305-330.

[M3] - An operator $L=a I-D_{t}^{j} D_{x}^{-j-\alpha}-D_{t}^{-j} D_{x}^{j+\alpha}$ and its nature in Gevrey functions, Tsukuba. J. Math., 17 no.1 (1993), 85-98.

[MH] M. Miyake and Y. Hashimoto, Newton polygons and Gevrey indices for partial differential operators, Nagoya Math. J., 128 (1992), 15-47.

[R1] J. P. Ramis, Dévissage Gevrey, Asterisque, 59/60 (1978), 173-204.

[R2] - Théorèmes d'indices Gevrey pour les équations différentielles ordinaires, Mem. Amer. Math. Soc., 48 no.296 (1984). 
[S] G. Szegö, Beitrage zur Theorie der Toeplitzschen Formen, Math. Zeitschrift, 6 (1920), 167-202.

[W] C. Wagschal, Une généralization du problème de Goursat pour des systèmes d'équations intégro-différentielles holomorphes ou partiellement holomorpes, J. Math. Pures Appl., 53 (1974), 99-132.

[Yn] A. Yonemura, Newton polygons and formal Gevrey classes, Publ. Res. Inst. Math. Sci., 26 (1990), 197-204.

[Ys1] M. Yoshino, Remarks on the Goursat problems, Tokyo J. Math., 4 (1980), $115-130$.

[Ys2] - Spectral property of Goursat problem, Tokyo J. Math., 4 (1981), 55-71.

[Ys3] - On the solvability of Goursat problems and a function of number theory, Duke Math. J., 48 (1981), 685-696.

[Ys4] - On the solvability of nonlinear Goursat problems, Comm. Partial Differential Equations, 8 (1983), 1375-1407.

[Ys5] — An application of generalized implicit function theorem to Goursat problems for nonlinear Leray-Volevich systems, J. Differential. Equations, 57 (1985), 44-69.

M. Miyake:

Department of Mathematics

School of Science

Nagoya University

Nagoya 464-01

Japan

M. Yoshino:

Faculty of Economics

Chuo University

Higashinakano, Hachioji

Tokyo 192-03

Japan 